\title{
Optical wavefront detection: A beginner tutorial
}

\author{
Shuiqin Zheng, ${ }^{1, *}$ Redha H. Al Ibrahim, ${ }^{2, *}$ Tien Khee Ng, ${ }^{3}$ Boon S. Ooi ${ }^{4}$ \\ Photonics Laboratory, Division of Computer, Electrical, and Mathematical Sciences and \\ Engineering, King Abdullah University of Science and Technology (KAUST), Thuwal 23955-6900, \\ Kingdom of Saudi Arabia \\ Izhengshuiqin@163.com \\ ${ }^{2}$ redha.ibrahim@kaust.edu.sa \\ 3itienkhee.ng@kaust.edu.sa \\ 4boon.ooi@kaust.edu.sa \\ *Equal contribution
}

\begin{abstract}
This article is designed to help readers understand some typical wavefront detection techniques and gain a preliminary understanding of wavefront detection. In this tutorial, the wavefront definition used in wavefront technology is given and we divide wavefront detection technologies into qualitative and quantitative categories. In each category, we discuss some typical systems and provide simulation results. This tutorial comes with Matlab simulation codes that readers can use to simulate mentioned wavefront detection technologies on their computers. We hope that this tutorial provides readers with all the tools needed to understand and simulate the different wavefront technologies and modify them based on their needs and interest.
\end{abstract}

\section{Keywords}

Wave optics; wavefront; optical field; phase imaging.

\section{Menu}

1. Introduction

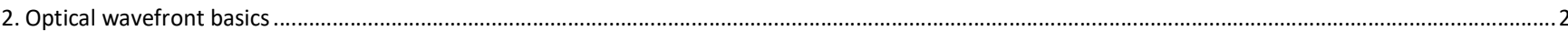

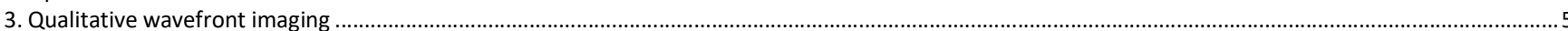

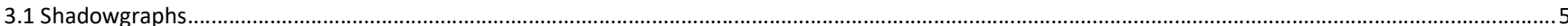

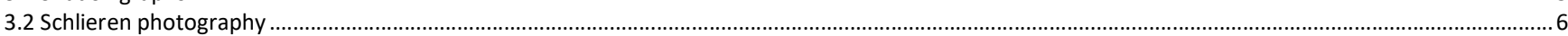

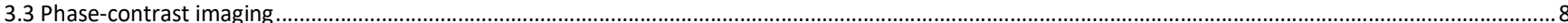

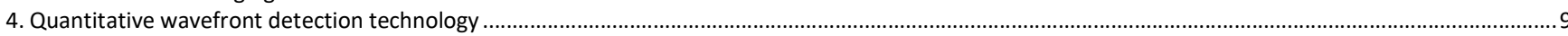

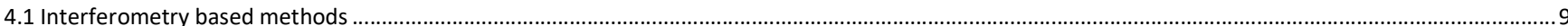

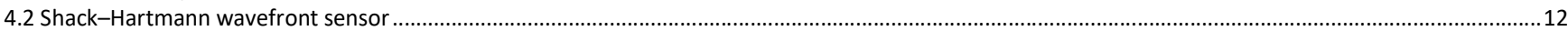

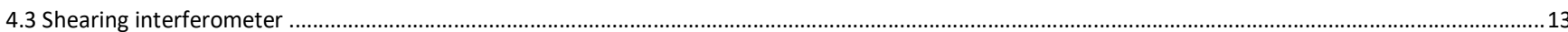

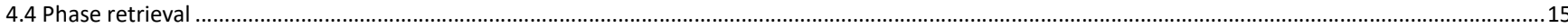

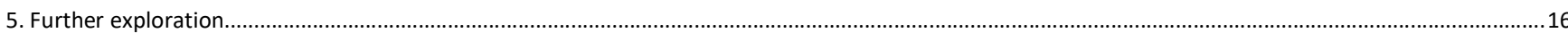

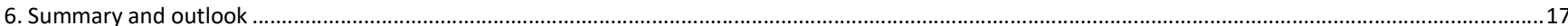

\section{Introduction}

A wavefront is a surface that connects points with the same phase within a wave and it represents characteristic information about the wavefield. Since light is an electromagnetic wave, the optical wavefront is an important characteristic of the optical field. Although optics is an ancient discipline and has made great progress, no available photoelectric detector is capable of directly measuring the phase of light. Therefore, measuring the phase of light is quite challenging. Despite this difficult challenge, several fields such as biomedical imaging, adaptive optics, and lens design can benefit substantially from phase detection.

In nature, many specimens do not exhibit sufficient light absorption in a certain spectral range. However, they do introduce different phase delays to the illuminating light and can be regarded as phase objects that only introduce phase modulation into the light $[1,2]$. Their non-uniform optical phase modulation can reflect the spatial distribution of the optical path difference. This is caused by a non-uniform refractive index or thickness. For such a transparent specimen, the amplitude distribution of the final detected optical field does not contain sufficient information or details, but the phase distribution does. For example, many cells such 
as protozoa, bacteria, sperm tails, and other cell structures are transparent without staining [3-5]. These cells absorb light weakly, so imaging them directly produces a low-contrast image, in which it is difficult to distinguish cell structures. However, the different densities and compositions of the observed specimen often cause different phase shifts in the light that passes through them. In the field of coherent diffraction imaging, the far-field diffraction pattern is used to reconstruct the optical field at the target position [6-8]. The main challenge is phase retrieval because phase information is inevitably lost during the measurement of diffraction patterns. Using prior knowledge or collection of multiple diffraction patterns, the lost phase distributions can be constrained and recovered. In the fields of adaptive optics and lens design, it is always desired to minimize and eliminate wavefront aberration as much as possible, to enhance image quality and increase resolution [9-11]. Therefore, measuring the aberrated wavefront is quite important. Moreover, the high-order modes of lasers have special wavefront structures, such as Laguerre-Gaussian beams and Hermitian-Gaussian beams [12-14]. Diagnosing the wavefront of this kind of structured light can help us to better understand and further study these types of laser beams. The above are our main motivations for developing phase- and wavefront detection technologies.

This article is intended to help readers understand some typical wavefront detection techniques and gain a preliminary understanding of wavefront detection. The content of this article has points related to Fourier optics. But as an entry-level tutorial, we try not to involve concepts in Fourier optics. Readers are advised to be familiar with Fourier optics for further in-depth research, as the Fourier domain provides a deeper understanding of wavefront behavior. This tutorial includes Matlab codes [15] to make it convenient for readers to simulate optical processes on their computers. We hope that readers can modify the parameters of simulations to understand how these parameters affect the results, and this way will help to build the readers' intuition to wavefront detection. Furthermore, readers are encouraged to build on the provided simulations and implement their own simulations to facilitate their needs and research goals. Readers are welcome to submit your code related to the non-included wavefront detection. Also, readers are encouraged to ask questions on the website [15] about code bugs, technical issues, and anything you want to know about wavefront detection. This will help to update this article and codes so as to help more readers.

\section{Optical wavefront basics}

In practical applications, it is important to pay attention to the wavefront of the single-frequency steadystate wave, in which the amplitude and the phase of each point do not change with time. A single-frequency steady-state wave can be expressed as

$$
u(\vec{r}, t)=A(\vec{r}) \cos \left(\omega_{0} t+\phi(\vec{r})\right)
$$

where $\vec{r}$ is the location vector and $t$ represents time. $\omega_{0}$ is the angular frequency of the field. Note that $\phi(\vec{r})$ is the spatial phase and $A(\vec{r})$ is the spatial amplitude, and both are functions only of $\vec{r}$. If the phase is set to equal to some constant as $\phi(\vec{r})=C$, a surface is constructed, and this surface is the equiphase surface of the optical field (wavefront). For convenience, a time-independent complex-values function is used to describe the single-frequency steady-state wave field. First, Euler's formula is used to rewrite Equation (1) as

or

$$
u(\vec{r}, t)=A(\vec{r}) \mathrm{e}^{i \phi(\vec{r})} \mathrm{e}^{i \omega_{0} t}+A(\vec{r}) \mathrm{e}^{-i \phi(\vec{r})} \mathrm{e}^{-i \omega_{0} t}
$$

$$
u(\vec{r}, t)=A(\vec{r}) \mathrm{e}^{i \phi(\vec{r})} \mathrm{e}^{i \omega_{0} t}+c \cdot c
$$

where $c . c$ is the complex conjugate of the front part. The complex conjugate is often ignored as it doesn't add any information when describing a field, and the field can be described as

$$
U(\vec{r})=A(\vec{r}) \mathrm{e}^{i \phi(\vec{r})}
$$

$U(\vec{r})$ is a complex-valued function of the location vector $\vec{r}$ and is independent of the time $t$. Because all points in the field have the same angular frequency $\omega_{0}$, The original physical field signal can be reconstructed using the complex-valued function $U(\vec{r})$ and the angular frequency $\omega_{0}$ via $u(\vec{r}, t)=$ $U(\vec{r}) \mathrm{e}^{i \omega_{0} t}+$ c.c. A monochromatic laser is an electromagnetic wave with a fixed frequency, a complexvalued function $E(\vec{r})$ can be used to model the electrical field of a uniformly polarized monochromatic laser. $E(\vec{r})$ satisfies the scalar Helmholtz equation in a vacuum [16], 
where $k_{0}=\omega_{0} / c$.

$$
\nabla^{2} E(\vec{r})+k_{0}^{2} \cdot E(\vec{r})=0
$$

Traditional wavefront definition targets an equiphase surface, but it is more important that the field distribution reaches a receiving plane, especially in wavefront detection. That is the amplitude and phase of each point on the receiving plane. The receiving plane can be photographic film, a photoelectric device, etc. In short, an optical disturbance that directly interacts with the material and components on the receiving plane is a two-dimensional complex-valued function $E(x, y)$. In this case, the spatial shape of the quiphase surface is not important as the field on the plane. And the corresponding complex-valued distribution on the plane can be expressed as

$$
E(x, y)=A(x, y) \mathrm{e}^{i \phi(x, y)}
$$

where $x, y$ are the coordinates in the Cartesian coordinate system on the plane. $A(x, y)$ and $\phi(x, y)$ are the amplitude and phase distributions on the plane. In general, this plane cannot be an equiphase surface. However, $E(x, y)$ is defined as the field function or generalized wavefront. The field function includes not only the amplitude distribution on the plane but also the phase distribution. In modern optics, the so-called wavefront refers to the optical field that directly interacts with the receiving plane. This generalized wavefront concept is different from the classical wavefront concept. For the modern development of wave optics, it is necessary to promote the wavefront concept in this way. At least this concept promotion can make us more rigorous in language. In the follow-up, the wavefronts mentioned are all two-dimensional complex-valued distribution on a plane. According to Equation (6), the wavefront can be divide into four classes. Below, $A_{c}$ and $\phi_{c}$ represent constants of amplitude and phase.

1. $A(x, y)=A_{c}, \phi(x, y)=\phi_{c}$, uniform collimated wavefront.

2. $A(x, y) \neq A_{c}, \phi(x, y)=\phi_{c}$, amplitude-only-modulated wavefront.

3. $A(x, y)=A_{c}, \phi(x, y) \neq \phi_{c}$, phase-only-modulated wavefront.

4. $A(x, y) \neq A_{c}, \phi(x, y) \neq \phi_{c}$, mixed-modulated wavefront.

For the first kind, it does not carry any spatial information. Usually, we use this wavefront as the light source for illuminating the specimen. For the second kind, the spatial information only exists in the amplitude distribution. This amplitude distribution is very easy to be measured because optoelectronic devices can respond to the light intensity, which is the square of the amplitude. Therefore, wavefront detection technology is not interested in the amplitude-only-modulated wavefront. The latter two are our targets. Obtaining planar two-dimensional optical phase distribution $\phi(x, y)$ is the basic goal of wavefront detection technology that traditional imaging systems cannot achieve.

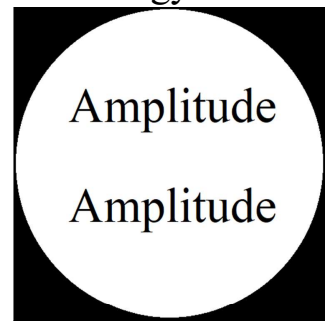

(a)Amplitude

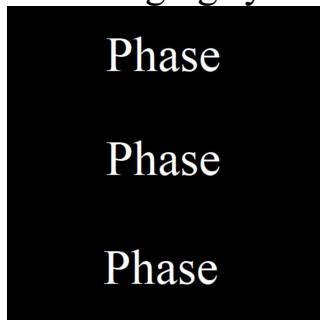

(b)Phase

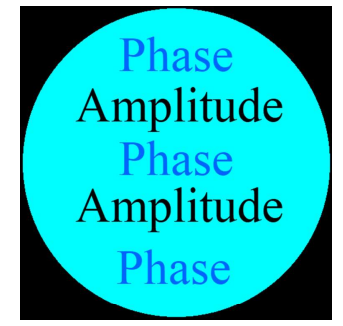

(c) Field

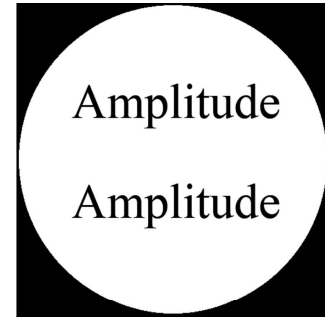

(d) Detected intensity

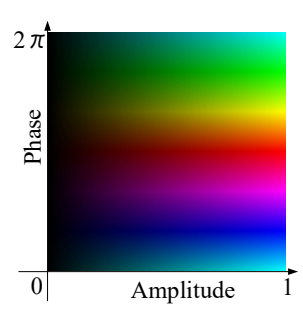

(e) Color bar

Fig. 1. (a) Amplitude distribution, where the black region represents 0 and the white region represents 1; (b) phase distribution, where the black region represents 0 and the white region represents $0.2 \pi$; (c) complex value distribution on the plane, where the brightness represents the amplitude and the color represents the phase; (d) the detected intensity distribution. (e) the color bar used in this tutorial for all figures representing complex value distribution.

First, it has to be made clear that phase distribution cannot be obtained by direct measurement of the light field, i.e. place the photoelectric sensor directly on the plane. An assumption can be made that there is an optical field whose amplitude and phase are shown in Fig. 1(a) and Fig. 1(b), respectively. The corresponding optical field is shown in Fig. 1(c), where the brightness represents the amplitude and the color represents the phase. This method will be used to draw the optical field later. And the color bar is shown in Fig. 1(e). If no statement is added, the same color bar will be used in such figures subsequently. Because the frequency of light vibration is so fast $\left(380 \sim 750 \times 10^{12} \mathrm{~Hz}\right)$, the detector cannot detect the phase of light (the fastest photodetector can only reach $\sim 100 \times 10^{9} \mathrm{~Hz}$ level). It can only respond to the intensity of 
light, which equals the square of the amplitude. Therefore, phase information is lost during measurement, as only the intensity on the plane can be obtained by a 2D-array photoelectric sensor, such as a CCD or CMOS camera. The intensity of the corresponding detected field (Fig. 1(c)) appears as Fig. 1(d). As can be seen, the 'Phase' information is lost. As mentioned, no available photoelectric detector can directly measure the phase of light. But the amplitude distribution of an optical field is much easier to measure than the phase distribution. Therefore, generally, wavefront detection technology couples phase information to the light intensity distribution and then demodulates out the phase distribution from the measured intensity distribution.

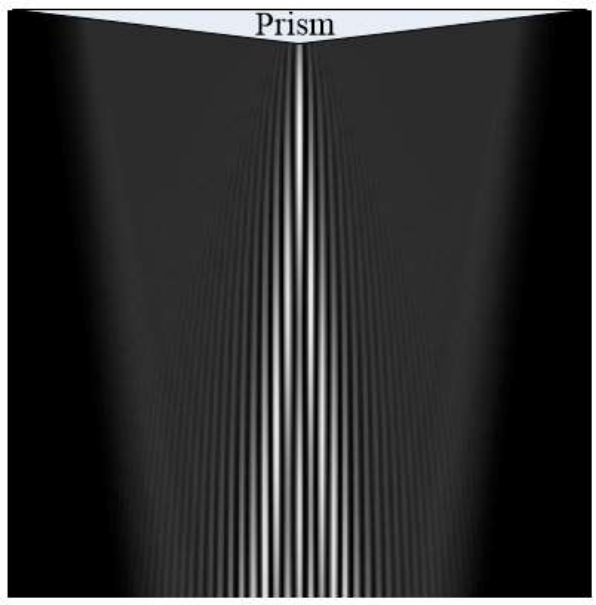

(a)

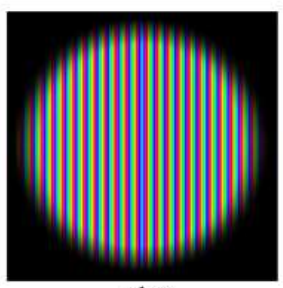

(b)

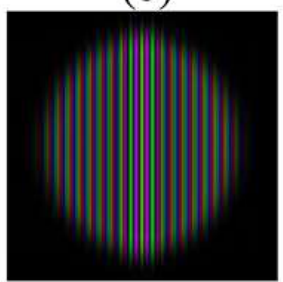

(d)

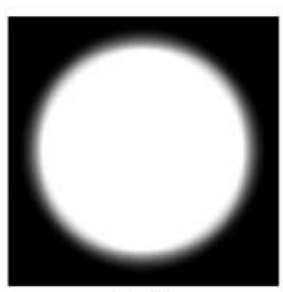

(c)

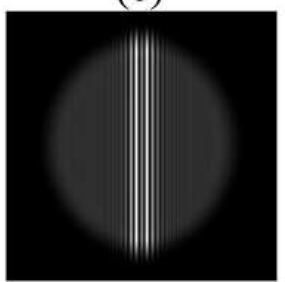

(e)

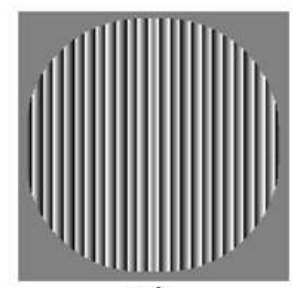

(d)

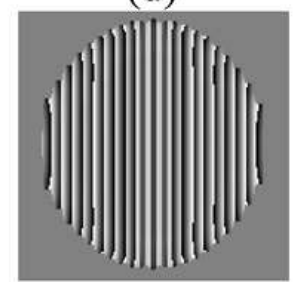

(f)

Fig. 2. (a) Propagation after parallel light illuminates a prism. The optical field just after leaving the prism: (b) field, (c) intensity, and (d) phase distributions. The optical field after propagation for some distance: (d) field, (e) intensity, and (f) phase distributions. In (b) and (d), the brightness represents the amplitude, and the color represents the phase.

Second, it is important to emphasize that the wavefront classification introduced in this article is for the light field distribution on a plane, rather than classify a light beam. The light beam can change from one type of wavefront to another type of wavefront during propagation. Figure 2 shows a simulation of propagation after a uniform parallel light beam illuminated a prism. And the wavefront that just leaves the prism can be regarded as a phase-only-modulated wavefront. However, during the propagation, the beam gets amplitude modulation, and the wavefront becomes a mixed-modulated wavefront. A given beam can have different amplitude and phase distributions at different propagation distances.

Uniform collimated wavefront is often used to illuminate. For the phase object, this results in a final interested wavefront with a uniform amplitude but a non-uniform phase distribution, which is exactly a phase-only-modulated wavefront. Therefore, many primitive wavefront detection technologies are used for this type of optical field. Then, the detected field degenerates to $A_{c} \mathrm{e}^{i \phi(x, y)}$. This amplitude distribution is a constant $A_{c}$ and does not contain any spatial information. The main information is carried on the phase term $\phi(x, y)$ and the amplitude is no longer important. In this case, the phase distribution $\phi(x, y)$ of the field function is also often named as wavefront in some other articles. In this tutorial, 'Field' refers to the generalized wavefront or optical field $E(x, y)$ that contains amplitude distribution and phase distribution. 'Intensity' refers to $|E(x, y)|^{2}=A^{2}(x, y)$, the intensity of the optical field. 'Amplitude' refers to $|E(x, y)|=A(x, y)$, the amplitude of the optical field. 'Phase' refers to $\phi(x, y)$, the phase of the optical field. If an optical field is represented by a letter, such as $E$, the corresponding amplitude, phase, and intensity distributions are expressed using subscripts of this letter, e.g., $A_{E}, \phi_{E}$, and $I_{E}$.

The main wavefront analyzed by primitive wavefront detection technology is the phase-only-modulated wavefront in which non-uniform phase information is loaded. In this case, we only need to focus on the measurement of the phase distribution. Of course, wavefront detection technology itself is not limited to phase measurement. It is useful that wavefront technology can measure both amplitude and phase distributions simultaneously. The wavefront that interests us may not contain information only in phase distribution. For example, Laguerre Gaussian beams and Hermitian Gaussian beams have special intensity distribution and phase distribution. Moreover, the amplitude and phase of the optical wave are also coupled 
simultaneously during propagation. This results in changes to both the amplitude and phase. Construction of complete light information requires both amplitude and phase information. Given a homogeneous medium, the amplitude and phase on any plane can be calculated using the information on the measured plane. Therefore, the ability to measure the amplitude and phase distributions simultaneously is useful in complex situations. This is why, in the field of optical wavefront detection, the concept of a wavefront has transitioned from an equiphase surface to a two-dimensional phase distribution on the detection plane, and even further to a complex value distribution on the detection plane, which includes amplitude and phase distributions. As the concept has been upgraded, the corresponding wavefront technology also improved.

Finally, it is critical to note that the target detected via wavefront technology is the wavefront of an optical beam on a certain plane. However, the information of the specimen can be detected using wavefront detection. This is because illuminating the specimen using the optical beam causes specimen information to be loaded into the beam. Therefore, in wavefront detection, what is detected is the phase information and even the amplitude information of the optical beam on a certain plane.

\section{Qualitative wavefront imaging}

Wavefront detection can be divided into qualitative and quantitative wavefront imaging. Qualitative wavefront imaging is a visualization technique. Its purpose is to directly reflect the phase information on the light intensity distribution so that the human eye can directly observe the phase distribution. However, this technique cannot produce a quantitative phase distribution from the detected image. This type of technology is aimed at an optical field with a uniform intensity distribution but an uneven phase distribution. If the intensity is not uniform, the visualized phase distribution is superimposed on the original amplitude distribution, which makes it impossible to distinguish between amplitude and phase distributions of the input field. Quantitative wavefront imaging is often a computational imaging technique. Its raw data is not intuitively understood and the human eye cannot directly see the phase distribution from the raw data. By processing the data, the phase and amplitude distribution can be reconstructed. The quantitative phase measurement provided by this technology can be used to calculate the optical path difference that is caused by a non-uniform refractive index or thickness. Being able to measure the amplitude and phase distributions simultaneously is useful in complex situations.

In this chapter, we introduce some typical qualitative wavefront imaging technology. Qualitative wavefront imaging technology is able to determine whether the phase of the wavefront has a non-uniform distribution and where the distribution is non-uniform. Qualitative wavefront imaging is able to display invisible phase information. It is a real-time, intuitive optical visualization technology. The goal of qualitative wavefront imaging technology is to reveal phase information from a beam that is uniform in amplitude but uneven in phase. Therefore, this type of technology could be referred to more precisely as qualitative phase imaging.

\subsection{Shadowgraphs}

A shadowgraph is the simplest qualitative phase imaging technology [17-19]. After a uniform collimated wavefront illuminates the specimen, the light beam enters an ordinary imaging system. This technology makes the photodetector or sensor of the imaging system deviate from the ideal imaging plane of the specimen in order to achieve quantitative phase imaging, as shown in Fig. 3. Figure 4 shows a simulation of the system. Figure 4(a) is an input optical field, where the brightness represents the amplitude and the color represents the phase. The 'Phase' is a region of $0.2 \pi$ phase modulation. If no additional instructions are added, this field is used as an input to subsequent simulations of the wavefront detection technologies in this tutorial. Figure 4(b) shows how the 'Phase' modulation causes an intensity change when the sensor deviates from the ideal imaging plane. As shown in Fig. 4(c), when the sensor is located on the ideal imaging plane, the image is a low-contrast-intensity image of the word 'Phase'. However, the word 'Phase' starts to appear when the defocusing distance increases. This is because the movement of the sensor or image plane corresponds to the movement of the object plane. The non-uniform phase distribution causes the phase to be coupled to the light intensity, causing the light intensity to change during propagation. This means that the sensor no longer measures the light intensity distribution when leaving the object, but the actual object 
plane is a certain distance away from the specimen. Because it must deviate from the ideal imaging plane to obtain obvious intensity variation, the in-focus object plane is no longer on the specimen and the resolution of this technology is relatively low. Therefore, this technique is often used for macro wavefront disturbances or other applications that do not need high resolution, such as detection of a difference in temperature or gas, or a shock wave in transparent air.

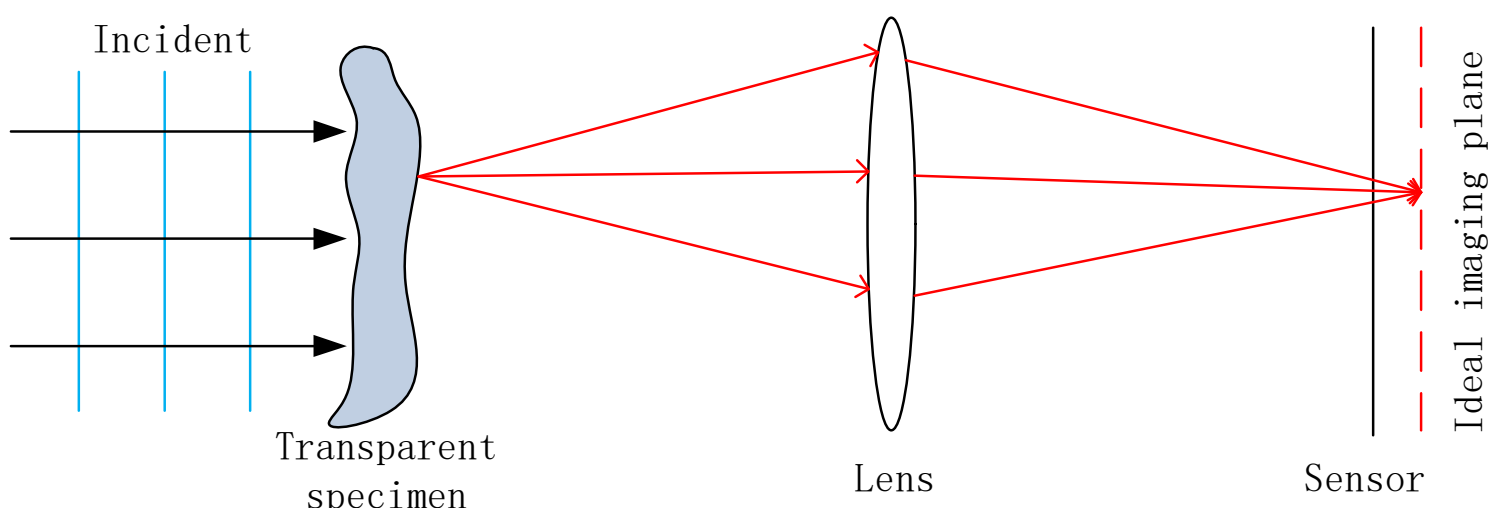

Fig. 3. The shadowgraph system.

(a)

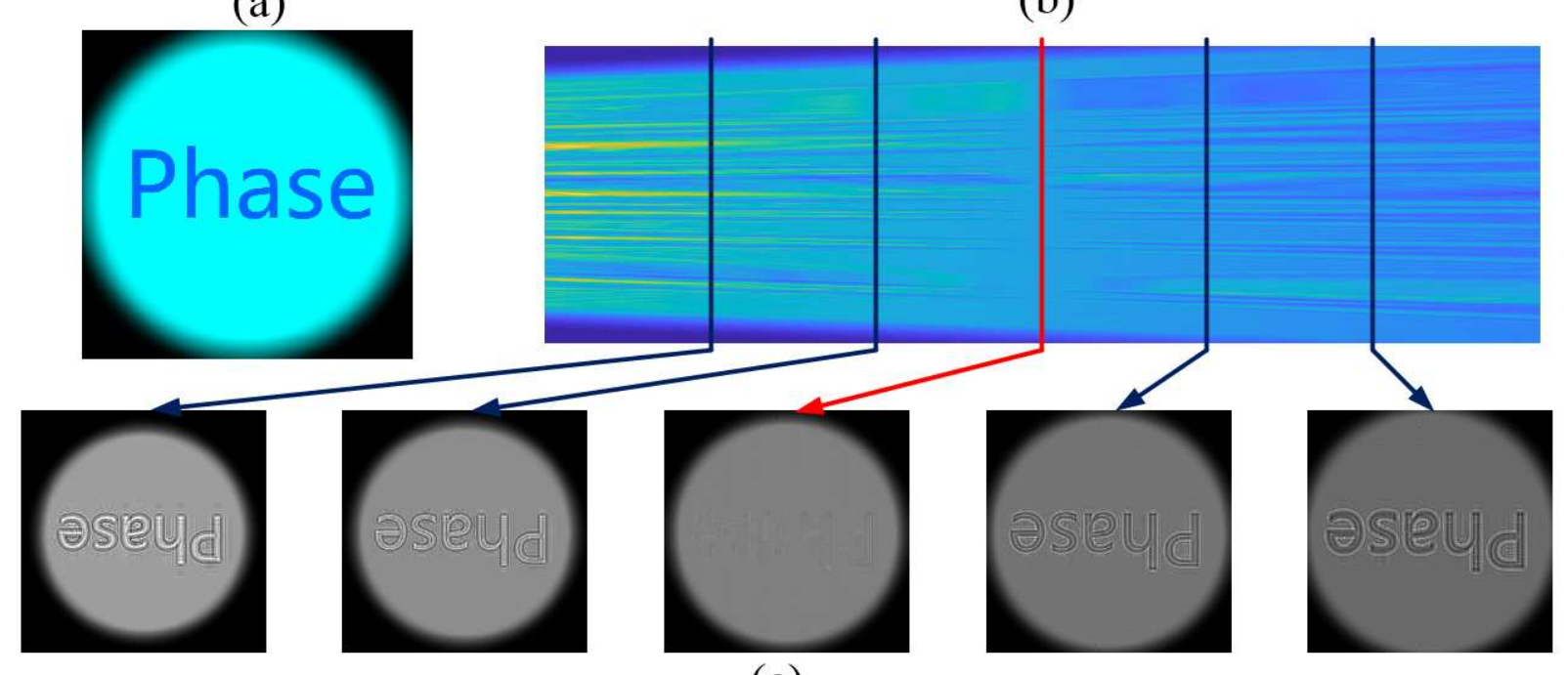

(c)

Fig. 4. Simulation of a Shadowgraph system: (a) the input field, where the brightness represents the amplitude and the color represents the phase; (b) the imaging intensity change along the propagation direction near the ideal image plane (the red line is the ideal imaging plane); and (c) the detected image near the ideal image plane.

\subsection{Schlieren photography}

Schlieren photography is another wavefront imaging technology for macro wavefront disturbances which is used to capture flows of fluids of varying densities [20-22]. It is widely used in aeronautical engineering to photograph the flow of air around an object. The classical implementation of an optical Schlieren system uses a collimated source to illuminate the specimen. A knife-edge is placed at the focal point, positioned to block about half of the light, as shown in Fig. 5. Wavefront variations caused by density gradients in the fluid distort the collimated light beam. This distortion creates spatial variation in the intensity of the light and qualitatively displays the distorted wavefront. With a collimated wavefront or a uniform intensity distributed parallel light, this simply makes the photograph half as bright. However, with a wavefront disturbance, the distorted beam focuses imperfectly, and parts that have been focused in an area covered by the knife-edge are blocked. The result is a set of lighter and darker patches that correspond to 
positive and negative wavefront gradients in the direction normal to the knife edge. The final image measures the first derivative of density in the direction of the knife edge.

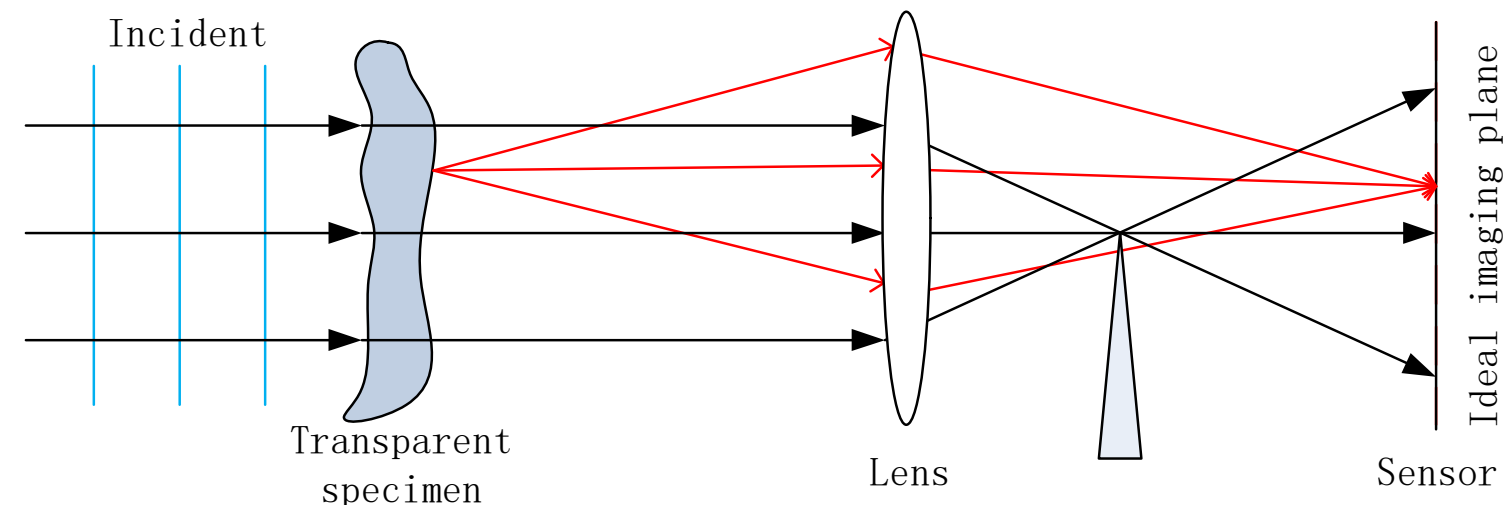

Fig. 5. The Schlieren photography system

Figure 6 shows a simulation of a shadowgraph system. Without a knife-edge blocking the beam, the imaging plane shows a low-contrast image. However, when the knife-edge starts to block the beam and moves horizontally, it causes 'Phase' to begin to appear. The intensity contrast is highest when half of the focal beam is blocked. At this time, the image is blurred in the horizontal direction. If most of the focused spot is blocked, only a small portion of the light passes. This eventually becomes a dark field image and finally shows the edge of a horizontal phase change. However, if the knife edge is rotated by 90 degrees and moved vertically, the highest contrast image is blurred in the vertical direction and a dark image that shows the edge of a phase change along the vertical direction, as shown in Fig. 7. Since the sensor is on the imaging plane, the detailed wavefront information is high-resolution. However, obstruction of the knife-edge causes an additional blur to be added. By appropriately controlling the position of the knife-edge, the blur can be controlled. Therefore, this method offers a higher resolution than the shadowgraph.
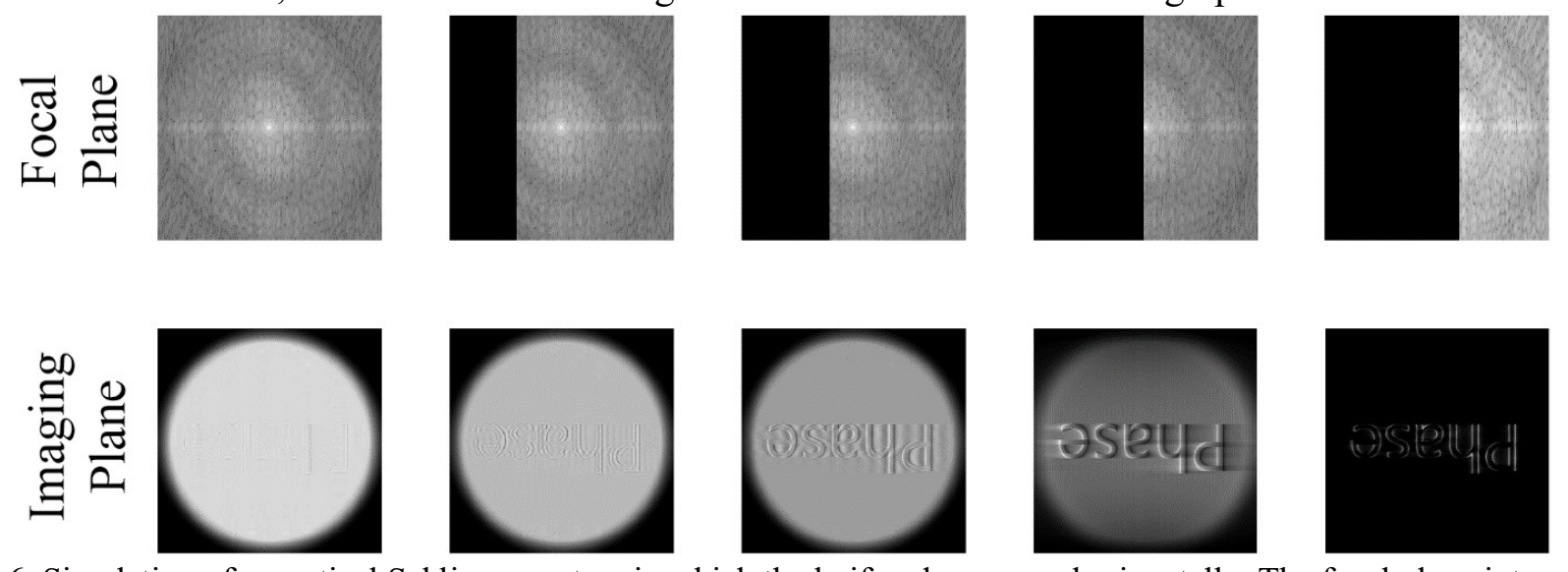

Fig. 6. Simulation of an optical Schlieren system in which the knife-edge moves horizontally. The focal plane intensity is on a $\log$ scale.
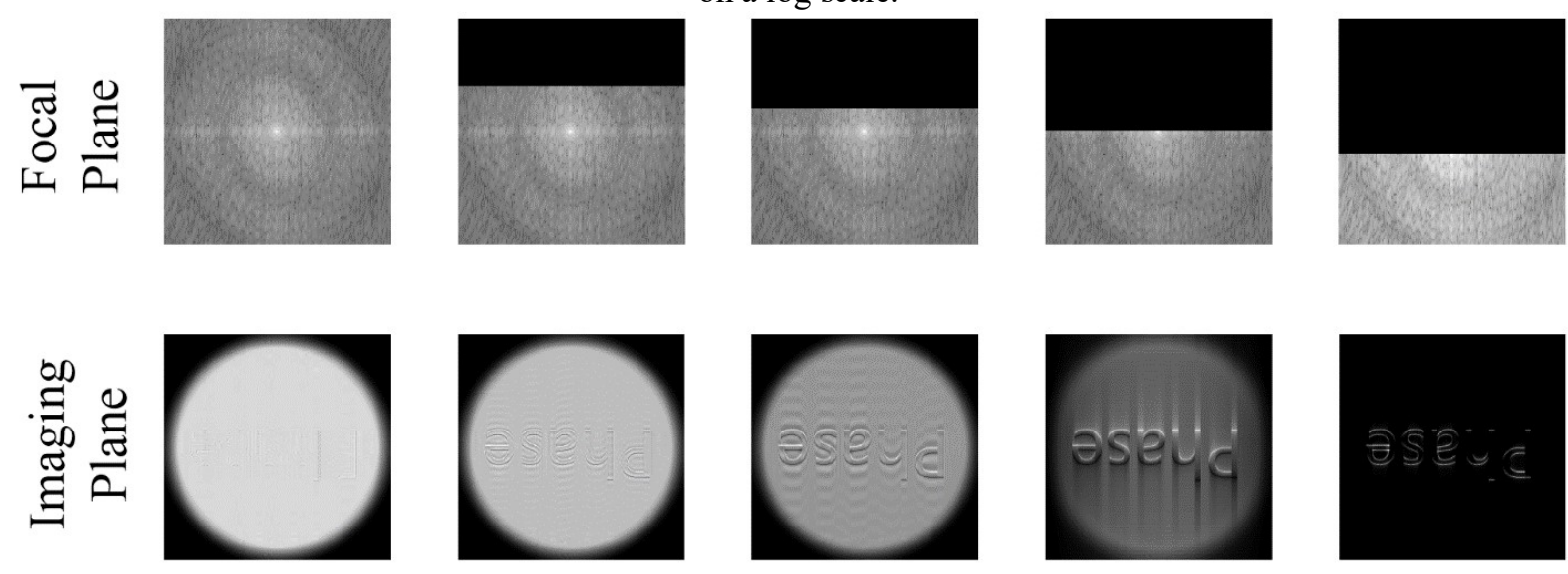
Fig. 7. Simulation of an optical Schlieren system in which the knife-edge moves vertically. The focal plane intensity is on

\subsection{Phase-contrast imaging}

a $\log$ scale.

Phase-contrast imaging is a method of qualitative wavefront imaging that is commonly used in bioimaging [23-25]. The basic phase-contrast imaging system requires a phase plate with a quarter-wave phase delay placed at the imaging point of the light source. When a parallel light illuminates a phase object, the wavefront that leaves the object can be described as $A \mathrm{e}^{i \phi(x, y)}$. The amplitude here is a constant, and thus the light intensity is equal everywhere. This means that the light intensity is weakly modulated. If $\phi(x, y) \ll$ 1 , then the wavefront can be described as $A(1+i \phi(x, y))$ according to Taylor's expansion formula. If a quarter-wave phase shift is performed on the latter terms, it becomes $A(1 \pm \phi(x, y))$, where '+' and '-' indicate positive and negative phase-contrast imaging, respectively. Thus, the phase change is displayed via the light intensity. Due to the presence of phase disturbances, the disturbed light has a direction that is inconsistent with the source of illumination. Thus, it focuses on another location in the focal plane. The undisturbed illumination light is focused on the center, while disturbed light is focused away from the center. Therefore, phase-contrast imaging can be achieved by placing a circular quarter phase plate at the focal point. The phase modulation difference between the inside and outside of the circle is a quarter-wave phase shift, as shown in Fig. 8. An additional positive quarter phase shift can be introduced to the undisturbed or disturbed light component. Finally, the phase changes are displayed as amplitude changes. Figure 9 shows a simulation of positive phase-contrast imaging. As the radius of the circle decreases, more information from the word 'Phase' is shown. Figure 10 shows a simulation of negative phase-contrast imaging. From these two simulations, it can be seen that as the circle becomes smaller, the outer circle includes more undisturbed illumination light introduced by the phase change, which makes the corresponding intensity distribution show more information from the word 'Phase'.

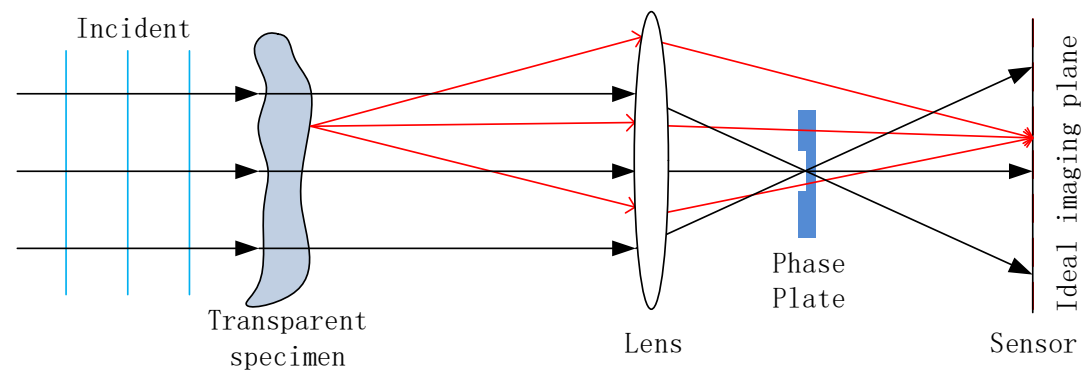

Fig. 8. The phase-contrast imaging system.
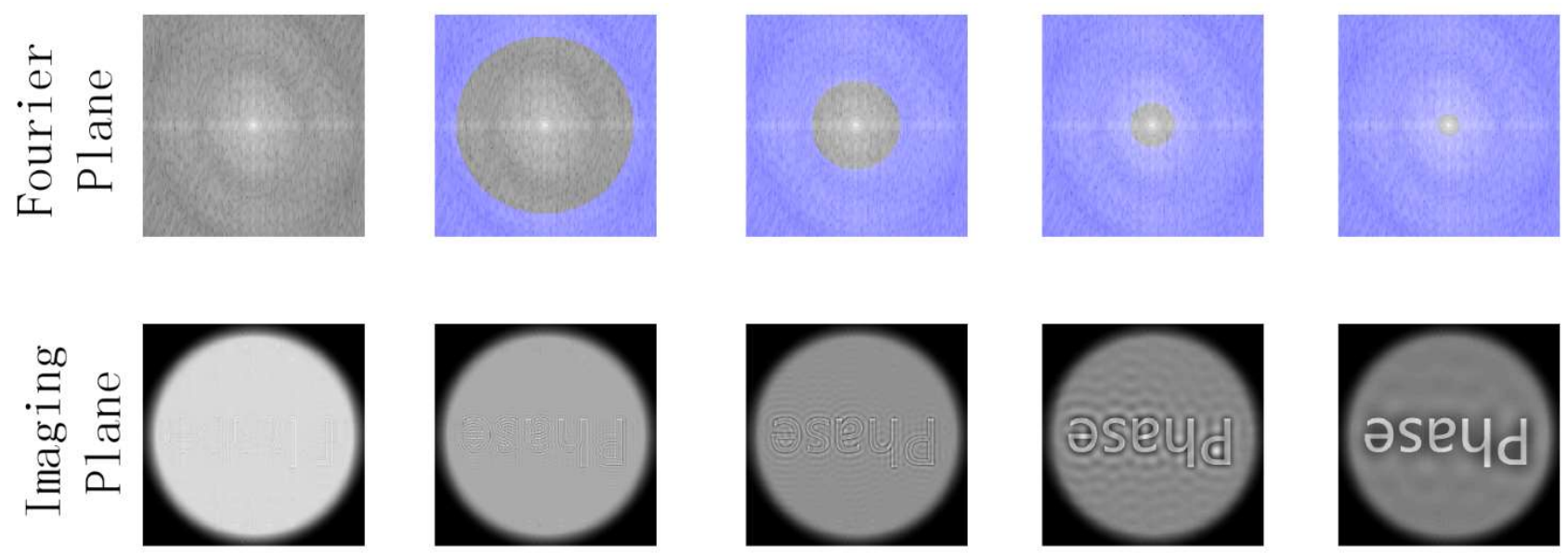

Fig. 9. A simulation of positive phase-contrast imaging. The focal plane intensity is on a log scale. The blue area indicates an additional quarter-wave phase shift. 

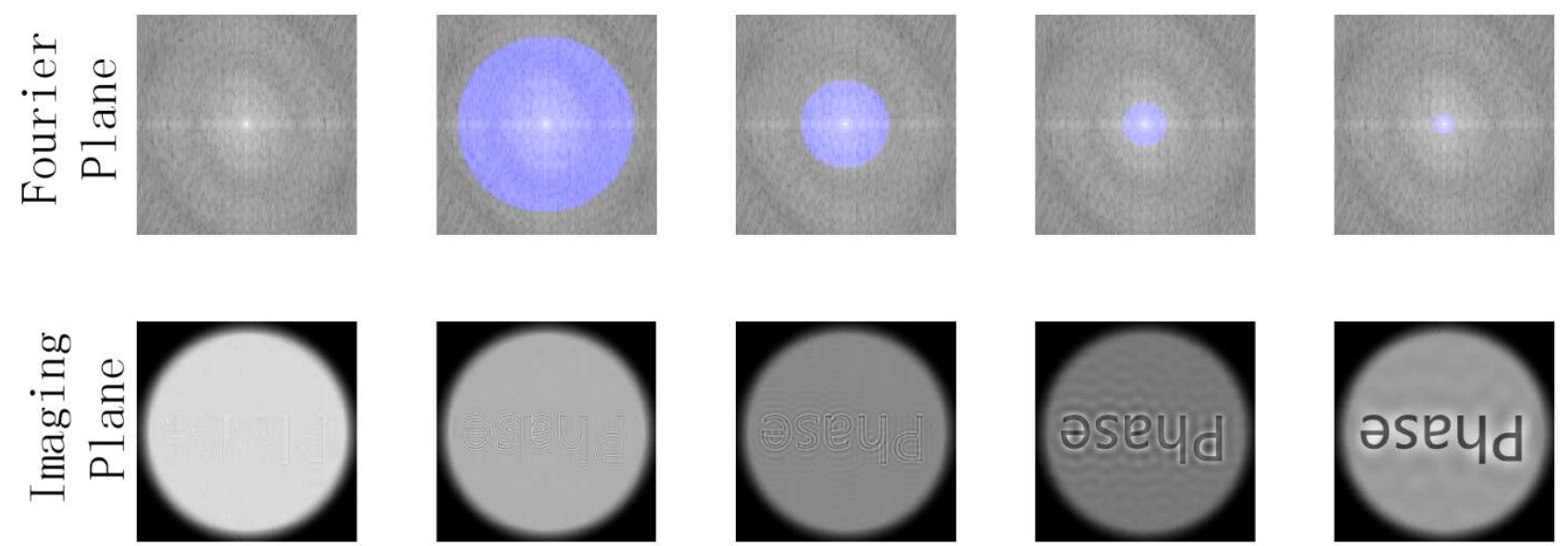

Fig. 10. A simulation of negative phase-contrast imaging. The focal plane intensity is on a log scale. The blue area indicates an additional quarter-wave phase shift.

\section{Quantitative wavefront detection technology}

Qualitative wavefront detection of phase objects can provide information regarding the position of the phase object. Qualitative phase imaging transforms the phase distribution into light intensity information. This requires that the amplitude distribution of the optical field to be measured is uniform or slowly changing. If it is not uniform, the visualized phase distribution is superimposed on the original amplitude distribution, which makes distinguishing between the two distributions impossible. Figure 11 shows an example of phase-contrast imaging of an input field with both amplitude and phase modulation, as shown in Fig. 11(a). The final detected image is as shown in Fig. 11(b). In Fig. 11(b), it is impossible to distinguish what information comes from the amplitude distribution versus the phase distribution. Therefore, qualitative phase imaging cannot measure the amplitude and phase distributions simultaneously. To solve this problem, a system that can measure both amplitude and phase is needed. Thus, quantitative wavefront detection is required to achieve quantitative phase detection. In the following sections, we will introduce some typical quantitative wavefront detection technology.
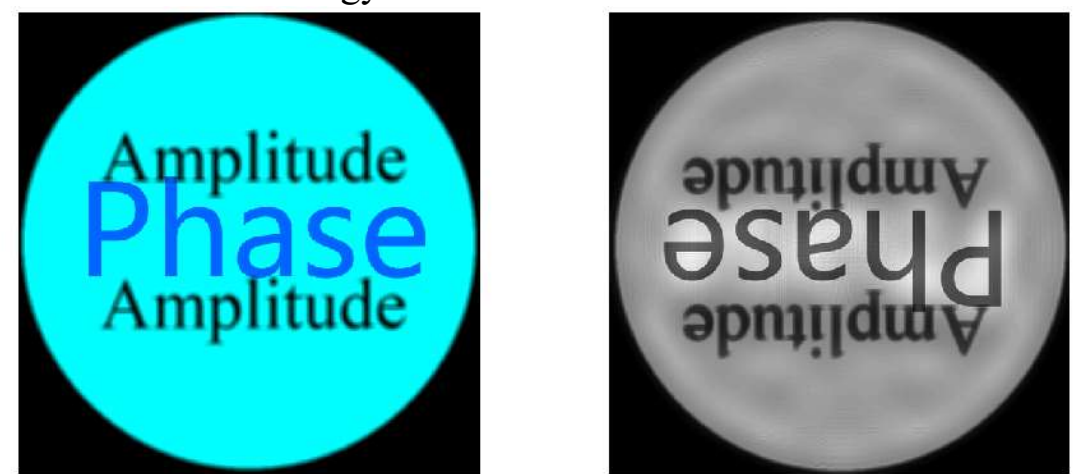

Fig. 11. An example of phase-contrast imaging of an input field with both amplitude and phase modulation: the (a) input field and (b) final detected image.

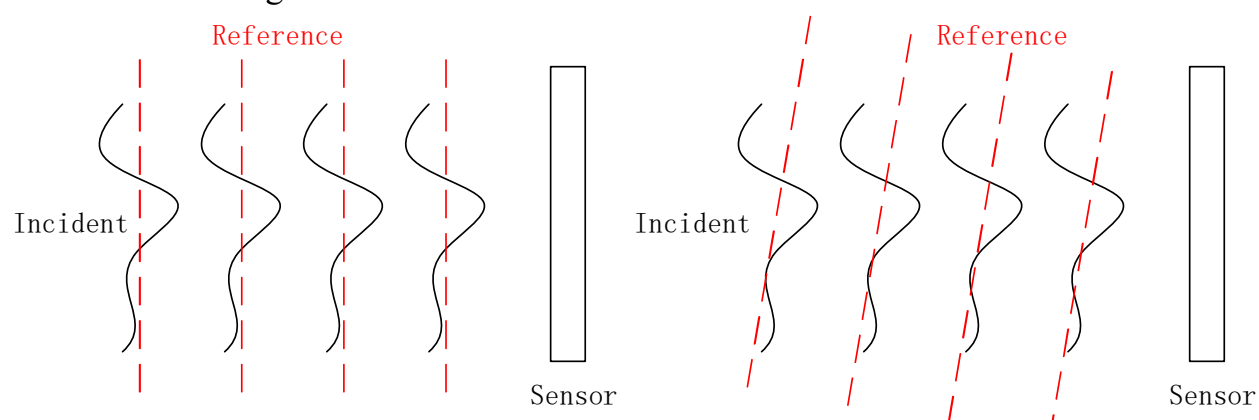

Fig. 12. On- and off-axis interference.

\subsection{Interferometry based methods}


The interference method is the most basic and high-precision wavefront measurement method [26-29].

This method uses a coherent light source that is split into two beams: the field $E$ to be measured and field $R$ whose wavefront is known.

$$
F(x, y)=E(x, y)+R(x, y)
$$

$F(x, y)$ is the final field on the detection plane. However, the detector generally can only respond to the optical intensity. The optical intensity signal received by the detector is

$$
I_{F}(x, y)=I_{E}(x, y)+I_{R}(x, y)+2 A_{E}(x, y) A_{R}(x, y) \cos \left(\phi_{E}(x, y)-\phi_{R}(x, y)\right)
$$

$R$ is a plane wave along the same direction as $E$, and can be expressed as $A_{R} e^{+i \phi_{R}}$, where $A_{R}$ and $\phi_{R}$ are constants. In this case, the interference is on-axis interference. The optical intensity signal received by the detector is then

$$
I_{F}(x, y)=I_{E}(x, y)+I_{R}+2 A_{E}(x, y) A_{R} \cos \left(\phi_{E}(x, y)-\phi_{R}\right)
$$

A single intensity distribution is insufficient to solve for the $A_{E}(x, y)$ and $\phi_{E}(x, y)$ of each location (x,y). In order to solve for $A_{E}(x, y)$ and $\phi_{E}(x, y)$, A multi-step phase shift is needed. For example, with an N-step phase shift, the nth intensity distribution is produced by adding a phase shift of $\frac{2 \pi}{N} n$ to the wavefront of $E$ or $R$, then the $\mathrm{n}^{\text {th }}$ intensity distribution is then

$$
I_{F, n}(x, y)=I_{E}(x, y)+I_{R}+2 A_{E}(x, y) A_{R} \cos \left(\phi_{E}(x, y)-\phi_{R}+\frac{2 \pi}{N} n\right)
$$

where $\mathrm{n}=0,1 \ldots, \mathrm{N}-1$. Then $A_{E}(x, y)$ and $\phi_{E}(x, y)$ can be calculated as

$$
\begin{gathered}
\phi_{E}(x, y)=\phi_{R}-\arctan \frac{\sum_{n=0}^{N-1} I_{F, n}(x, y) \sin \left(\frac{2 \pi}{N} n\right)}{\sum_{n=0}^{N-1} I_{F, n}(x, y) \cos \left(\frac{2 \pi}{N} n\right)} \\
A_{E}(x, y)=\frac{1}{A_{R} N} \operatorname{sqrt}\left\{\left|\sum_{n=0}^{N-1} I_{F, n}(x, y) \sin \left(\frac{2 \pi}{N} n\right)\right|^{2}+\left|\sum_{n=0}^{N-1} I_{F, n}(x, y) \cos \left(\frac{2 \pi}{N} n\right)\right|^{2}\right\}
\end{gathered}
$$

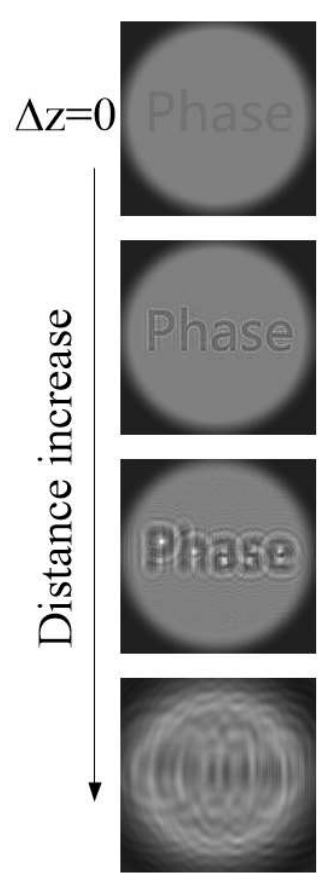

I1
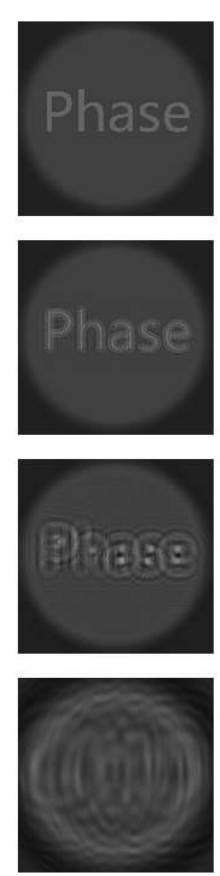

I2
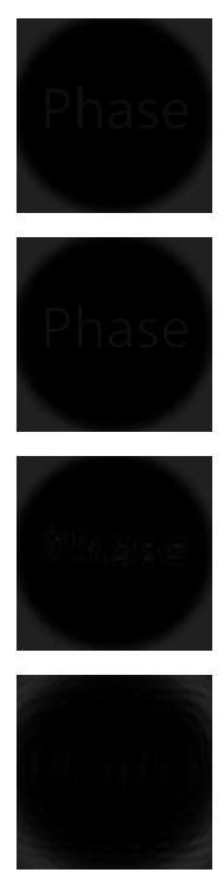

I3
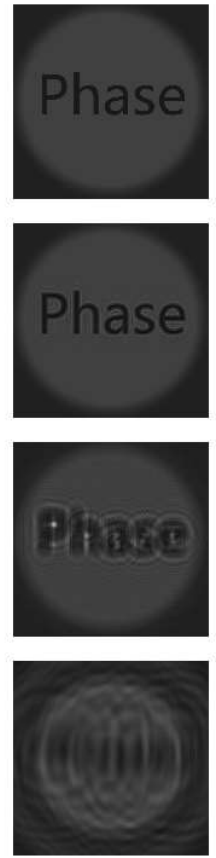

I4
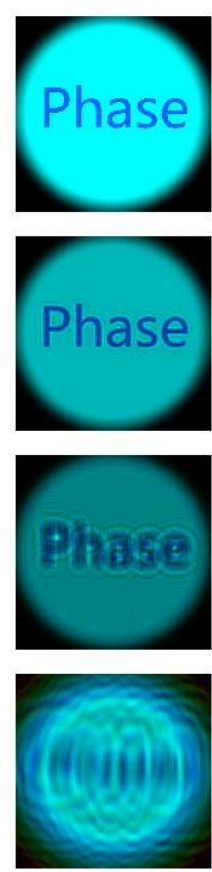

Detected Field
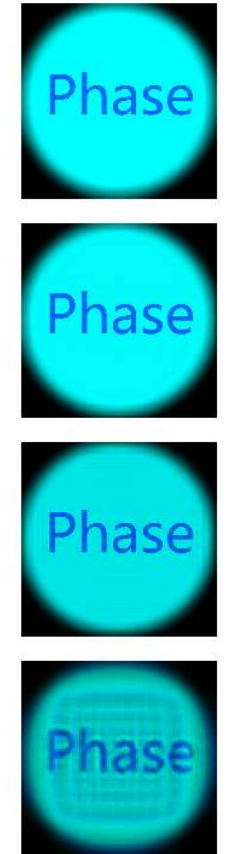

Backpropagated

field

Fig. 13. Simulation of 4-step phase-shift wavefront detection.

Figure 13 shows a simulation of wavefront reconstruction using a 4-step phase shift. The number on the left represents the distance between the input optical field and the detection plane or sensor. I1-I4 are the 
intensities captured on the detection plane. The detected fields are reconstructed from I1-I4, which correspond to the optical field on the sensor. The backpropagated fields are the calculated input fields through backpropagation from the detected field on the detected plane. When the input optical field is close to the sensor, the restored input optical field is accurate. However, the diffraction pattern from the rectangular aperture is superimposed at further distances. This is because when the distance is closer, most of the information is received by the receiving plane. However, as the distance increase further, not all information is received by the sensor, resulting in a strong deviation.

If the reference beam and the beam to be measured are directed at a certain angle from each other, the reference light is an inclined plane wave described by $A_{R} e^{+i k_{x} x}$. The optical intensity signal received by the detector is

$$
I_{F}(x, y)=I_{E}(x, y)+I_{R}+2 A_{E}(x, y) A_{R} \cos \left(\phi_{E}(x, y)-k_{x} x\right)
$$

A two-dimensional Fourier transforms can be used to extract AC (alternating current) terms using a bandpass filter. Then, the AC term can be expressed as

$$
I_{F, A C}(x, y)=A_{E}(x, y) A_{R} \exp \left\{i\left(\phi_{E}(x, y)-k_{x} x\right)\right\}
$$

$A_{E}(x, y)$ and $\phi_{E}(x, y)$ can be calculated from the AC term using

$$
\begin{gathered}
\phi_{E}(x, y)=\operatorname{angle}\left(I_{F, A C}(x, y)\right)+k_{x} x \\
A_{E}(x, y)=\frac{1}{A_{R}} \operatorname{abs}\left(I_{F, A C}(x, y)\right)
\end{gathered}
$$

Figure 14 shows a simulation of off-axis interference wavefront detection at various distances. The diffraction pattern of the rectangular aperture appears when the distance is far. This is also because not all information is received by the receiving surface when the distance is far. Figure 15 shows a simulation of off-axis interference wavefront detection using various angles. When the angle is large, the stripes are denser and the reconstructed optical field is clearer. This is because the reconstruction process requires the use of a band-pass filter and the maximum bandwidth of the band-pass filter is $k_{x} / 2$. When the bandwidth increases, more details become clearer. Therefore, off-axis interference requires the fringes to be as dense as possible. However, the upper limit of the fringe density is determined by the sampling density of the detector, i.e., the pixel size.

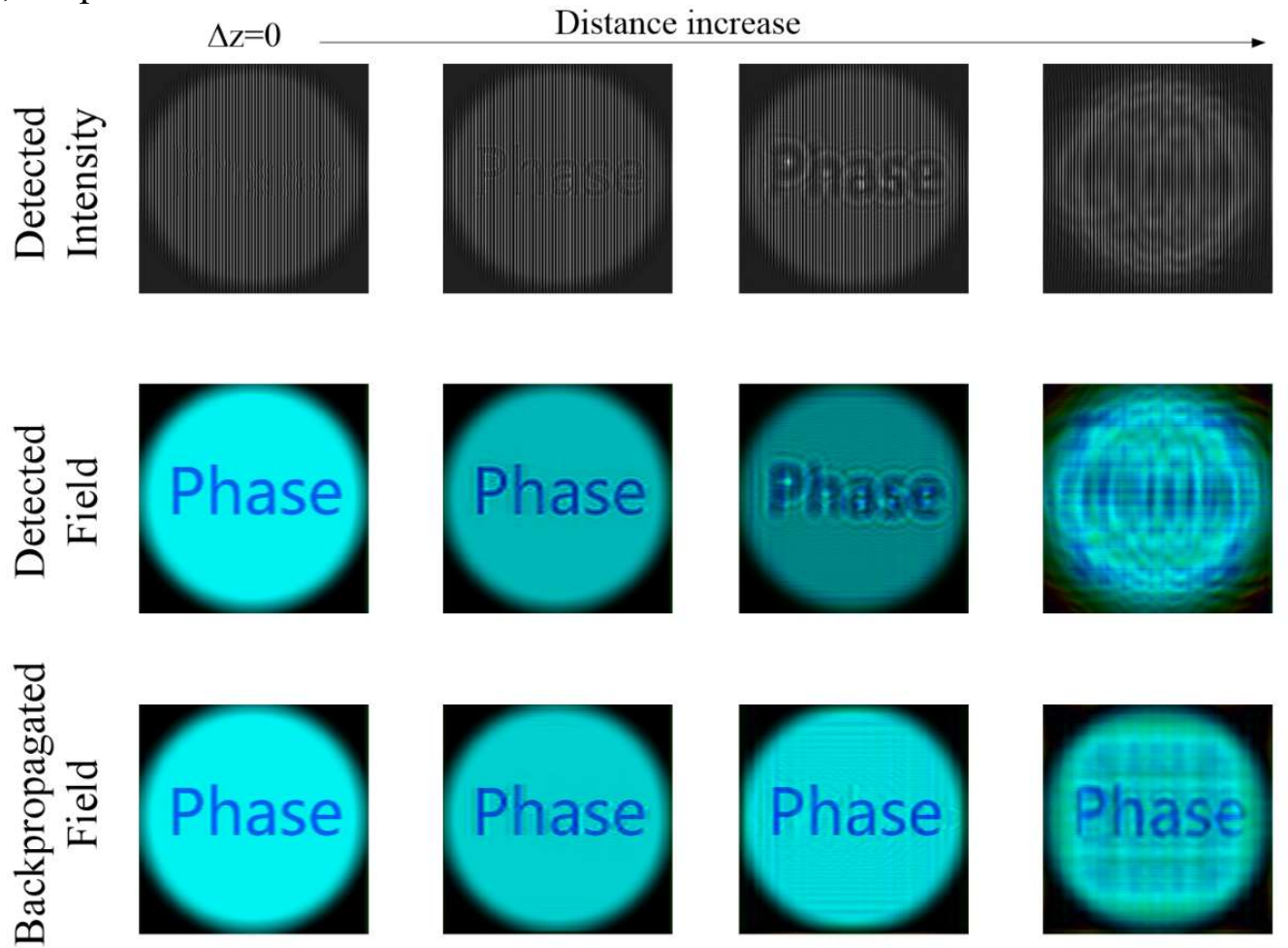

Fig. 14. Simulation of off-axis interference wavefront detection at various distances. 

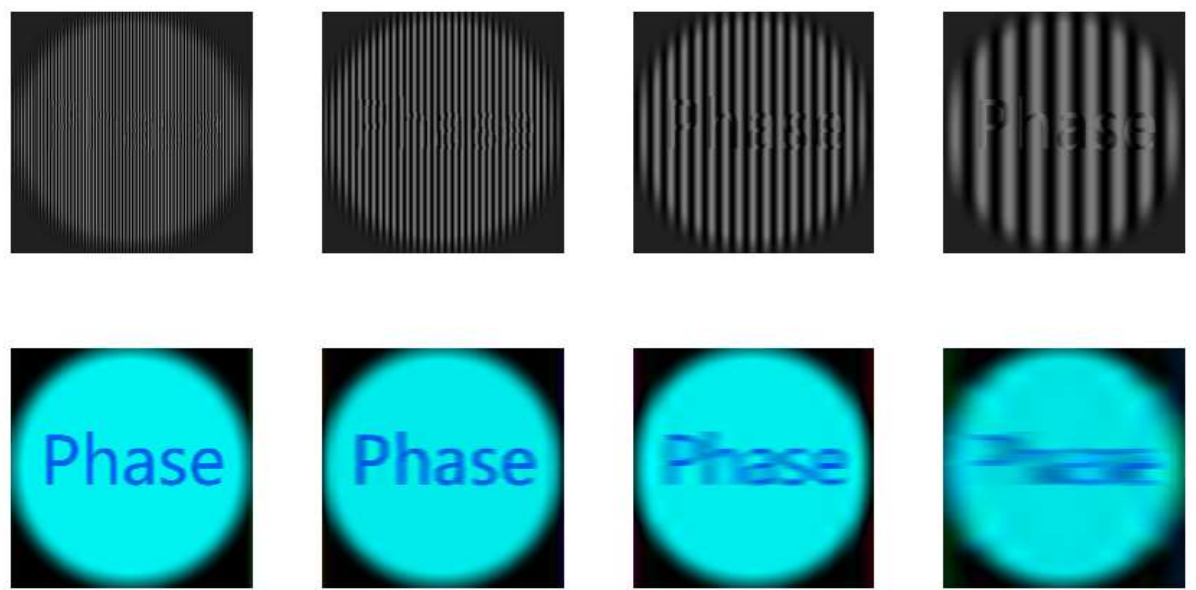

Fig. 15. Simulation of off-axis interference wavefront detection using various off-axis angles.

The interference method requires one coherent source split into two coherent beams. This requirement places some limitations on the method. The reference and measuring beams must originate from the same laser. After the two beams are separated, they pass through different optical paths. Instabilities in these optical paths, such as atmospheric disturbances, add error to the measurement. The system must be resilient against vibration. Therefore, although the interference method is simple and high precision, its antijamming ability is poor.

\subsection{Shack-Hartmann wavefront sensor}

The Shack-Hartmann wavefront sensor is commonly used in adaptive optics systems [30-33]. It consists of an array of lenses (each unit called lenslet) of the same focal length, as shown in Fig.16. Each lenslet divides the field into several inclined plane sub-waves and the phase gradient of the sub-waves across the lenslet is proportional to the displacement of the centroid. The relation between the phase gradient and displacement can be expressed as

$$
\left[\begin{array}{l}
\partial_{x} \\
\partial_{y}
\end{array}\right] \phi(x, y)=\frac{k_{0}}{f}\left[\begin{array}{l}
\Delta_{x} \\
\Delta_{y}
\end{array}\right]
$$

where $\Delta_{x}$ and $\Delta_{y}$ are the displacement of the centroid along the $\mathrm{x}$ - and $\mathrm{y}$ - directions, respectively, and $f$ is the focal length of the lenslet. The direct measurement data from this Shack-Hartmann wavefront sensor is the gradient distribution of the wavefront rather than the wavefront itself. However, the phase distribution can be reconstructed from the phase gradient using

$$
\phi_{R}(x, y)=F^{-1}\left\{-i \frac{f_{x} F\left\{\partial_{x} \phi(x, y)\right\}+f_{y} F\left\{\partial_{y} \phi(x, y)\right\}}{f_{x}^{2}+f_{y}^{2}}\right\}
$$

where $F$ and $F^{-1}$ are operators for Fourier transformation and Inverse Fourier transform, and $f_{x}, f_{y}$ are the spatial frequency after Fourier transform along $x$ - and $y$ - directions. Figure 16 shows simulated ShackHartmann wavefront detection using various numbers of lenslets. Obviously, more lenslets produce a higher spatial resolution. However, as the number of lenslets increases, the aperture of the sub-lens becomes smaller. When the focal length is constant, the dynamic range of the gradient shrinks. Of course, the focal length can be reduced, at the cost of reducing the sensitivity of the wavefront gradient. Therefore, there is a mutually restrictive relationship between the performance parameters of this wavefront sensor.

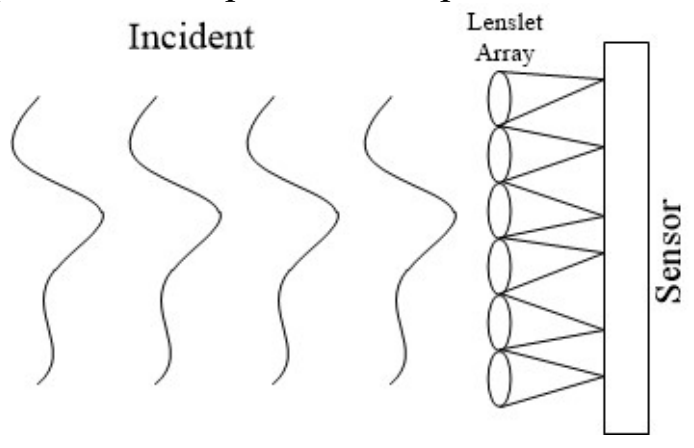


Fig. 16 The Shack-Hartmann system
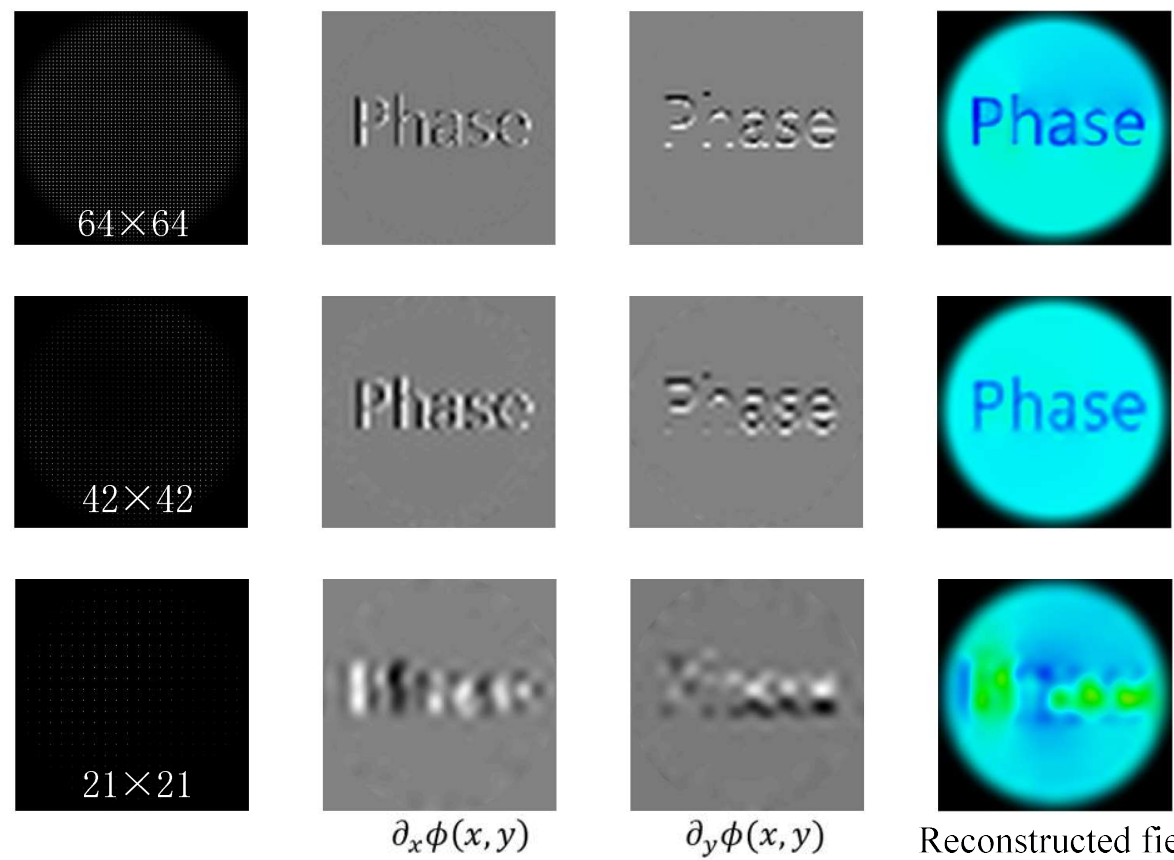

Reconstructed field

Fig. 17. Simulation of Shack-Hartmann wavefront detection using various numbers of lenslets.

\subsection{Shearing interferometer}

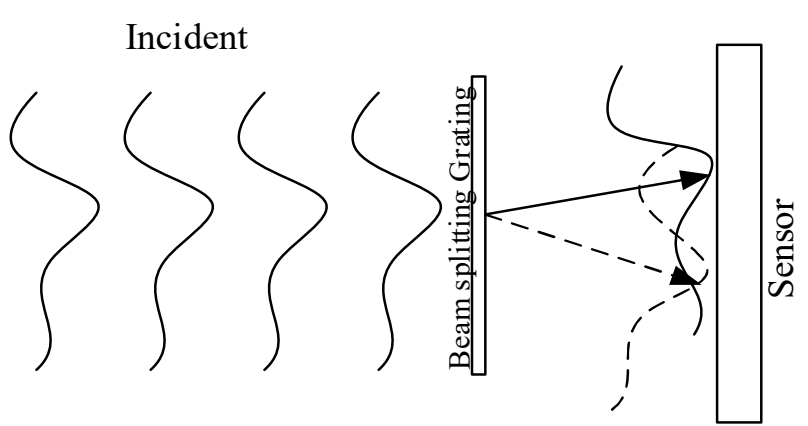

Fig. 18. Quadriwave lateral shearing interferometry.

The shearing interferometer is a simple means of observing wavefront disturbance and can be used to measure the phases of light beams [34-37]. It can solve the problem of poor anti-interference ability in the Interferometry based methods. Basic shearing interference splits the beam into two with displacement and tilting. If one beam exhibits displacement and tilting along the $\mathrm{x}$-direction and the other is the same as the input, then the detected field can be expressed as

$$
F(x, y)=E(x, y)+E(x+\Delta, y) \mathrm{e}^{-i k_{x} x}
$$

where $\Delta$ is the displacement and $k_{x}$ is the wave vector component in the x-direction. Then, the intensity distribution received is

$$
\begin{gathered}
I_{F}(x, y)=I_{E}(x, y)+I_{E}(x-\Delta, y)+I_{E}(x, y) \\
+2 A_{E}(x, y) A_{E}(x-\Delta, y) \cos \left(\phi_{E}(x, y)-\phi_{E}(x-\Delta, y)-k_{x} x\right)
\end{gathered}
$$

and the $\mathrm{AC}$ term is

However, $k_{x}$ is a design parameter that is known in advance, the whole term $-k_{x} x$ can be calibrated using the uniform collimated wavefront $A_{c} e^{+i \phi_{C}}$, and the AC term of the calibration intensity distribution will be

Then the whole term $-k_{x} x$ will be

$$
\left.I_{c, A C}(x, y)=A_{c} A_{c} \exp \left\{i\left(-k_{x} x\right)\right)\right\}
$$

$$
-k_{x} x=\operatorname{angle}\left(I_{c, A C}(x, y)\right)
$$


After calibration, the phase difference is calculated using

$$
\phi_{E}(x, y)-\phi_{E}(x-\Delta, y)=\operatorname{angle}\left(I_{F, A C}(x, y)\right)-\operatorname{angle}\left(I_{C, A C}(x, y)\right)
$$

when $\Delta x$ is small, then

$$
\phi_{E}(x, y)-\phi_{E}(x-\Delta x, y) \approx \Delta \frac{\partial \phi_{E}}{\partial x}
$$

Thus, the shearing interference method determines the phase gradient, much like the Shack-Hartmann method. To measure the gradient in the $\mathrm{x}$ - and $\mathrm{y}$ - directions, A quadriwave lateral shearing interferometry can be used [38], which splits the beam into four beams and produces two terms $\frac{\partial \phi_{E}}{\partial x}$ and $\frac{\partial \phi_{E}}{\partial y}$. It uses a diffraction grating to split the beam into four beams along four different directions, as shown in Fig. 18. Two of the beams are separated along the x-direction, while the other two are separated along the y-direction. The detected field can be expressed as

$$
F(x, y)=E\left(x-\frac{\Delta}{2}, y\right) \mathrm{e}^{i \frac{2 \pi}{T} x}+E\left(x+\frac{\Delta}{2}, y\right) \mathrm{e}^{-i \frac{2 \pi}{T} x}+E\left(x, y-\frac{\Delta}{2}\right) \mathrm{e}^{i \frac{2 \pi}{T} y}+E\left(x, y+\frac{\Delta}{2}\right) \mathrm{e}^{-i \frac{2 \pi}{T} y}
$$

The partial derivatives $\frac{\partial \phi_{E}}{\partial x}$ and $\frac{\partial \phi_{E}}{\partial y}$ can be calculated from the detected AC components in the horizontal and vertical directions from the receiving intensity distribution. If $\phi_{E}$ can be regarded as a field without a singularity, then $\phi_{E}$ can be reconstructed via the integration of these two terms. The phase distribution can be reconstructed from the wavefront gradient using

$$
\phi_{R}(x, y)=F^{-1}\left\{-i \frac{f_{x} F\left\{\partial_{x} \phi_{E}(x, y)\right\}+f_{y} F\left\{\partial_{y} \phi_{E}(x, y)\right\}}{f_{x}^{2}+f_{y}^{2}}\right\}
$$

Figure 19 shows a simulation of quadriwave lateral shearing interferometry with various displacements. A displacement that is too large or too small produces an inaccurate result. When the displacement is too small, the detected $\Delta \frac{\partial \phi_{E}}{\partial x}$ and $\Delta \frac{\partial \phi_{E}}{\partial y}$ is small and contains a lot of noise. When the displacement is too large, Equation (27) is no longer applicable and the result is inaccurate. The period of the grating is also quite important, because, as in the interference method, the density of the fringe period is proportional to the amount of information that can be extracted from the AC term. Therefore, a smaller period allows for a higher resolution, as shown in Fig. 20.

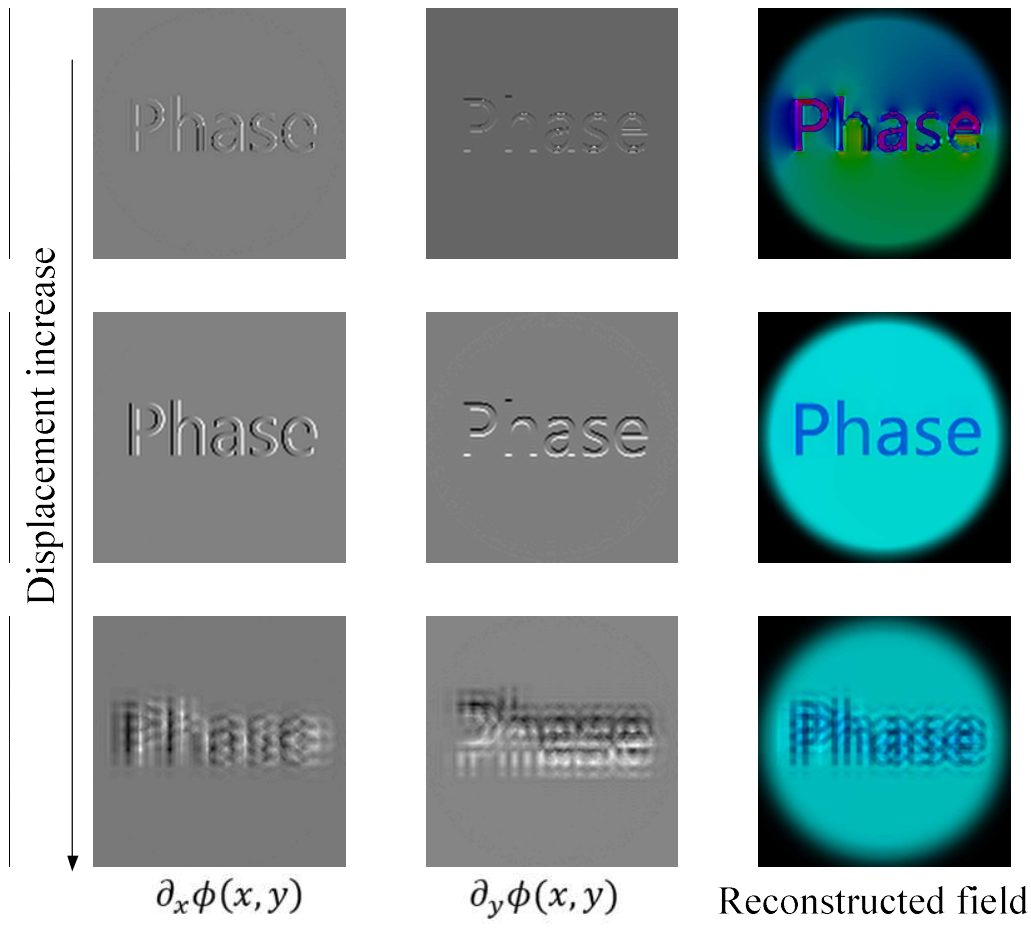

Fig. 19. Quadriwave lateral shearing interferometry with various displacements. 


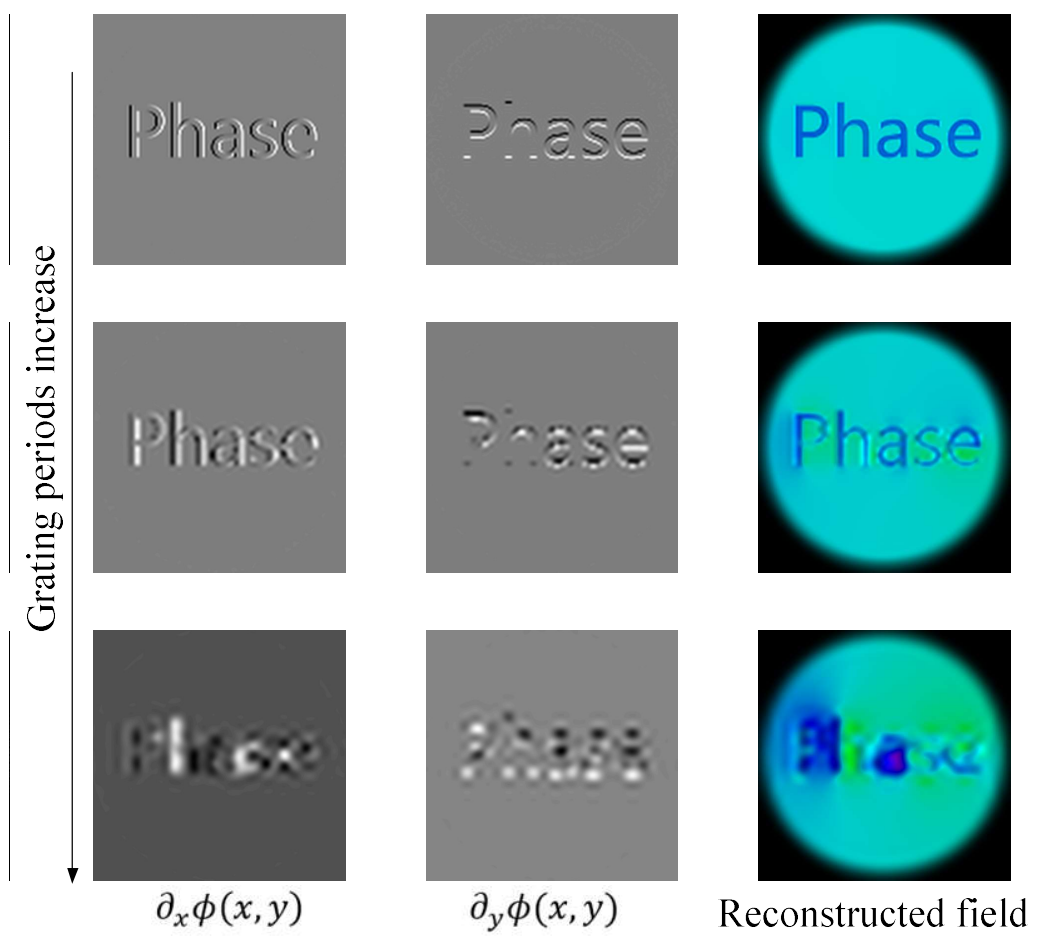

\subsection{Phase retrieval}

Fig. 20. Quadriwave lateral shearing interferometry with various grating periods.

Coherent diffractive imaging is a powerful tool for structure reconstruction and has been used to image biological cells, integrated circuits, and Nano-objects [39-42]. Without the need for an imaging system, image information can be extracted from diffraction patterns of a target that are produced using coherent sources. The essence of coherent diffractive imaging is an inverse source problem constrained by a wave equation and diffraction patterns. Since the phase distribution affects the far-field, the captured far-field intensity distribution can be used as a constraint on the phase distribution. Suppose that we obtain the light intensity distribution of the input field and the diffraction distribution of the far-field. When there is a certain phase distribution of the input field that makes the corresponding diffraction pattern consistent with the measured diffraction pattern, then this phase distribution is the phase of our incident field. The process can be done using a phase retrieval algorithm. Thus, the entire optical field can be reconstructed, including the amplitude and phase distributions.

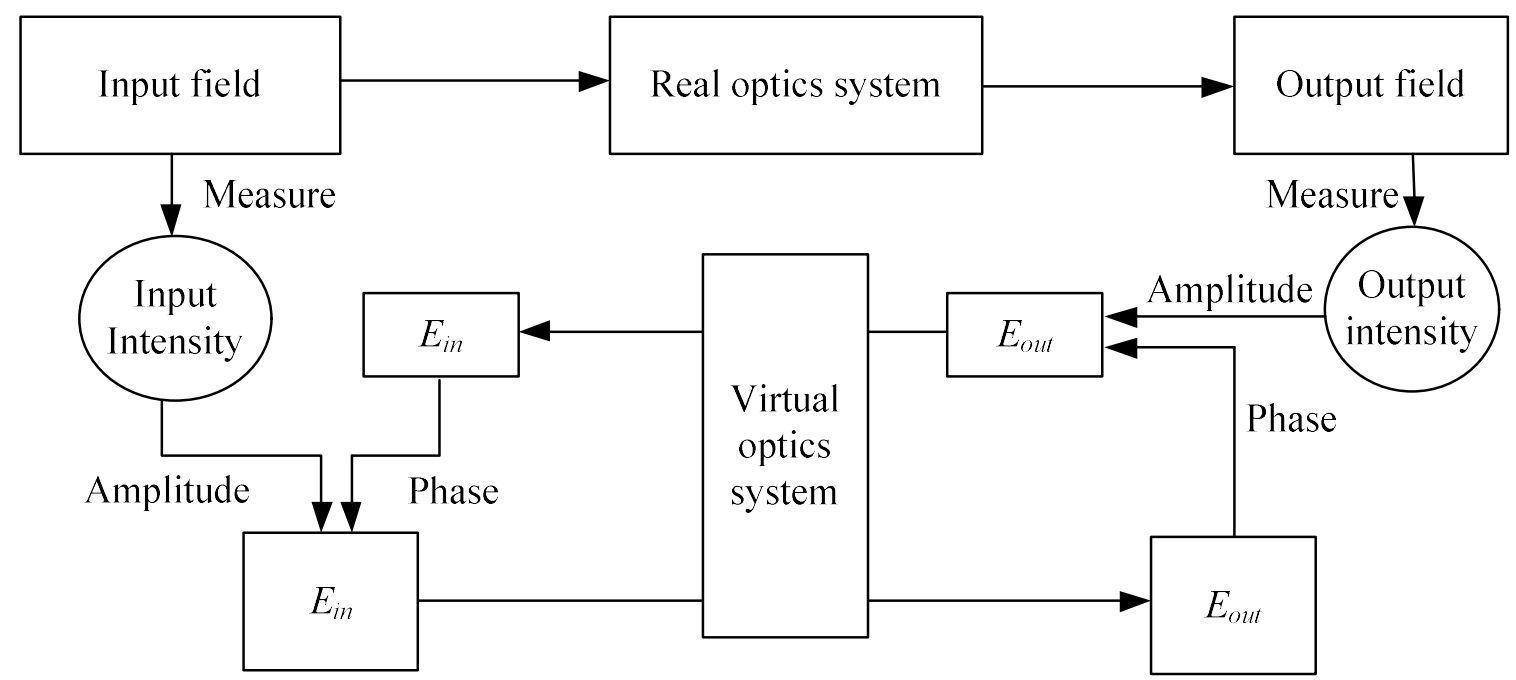

Fig. 21. Generalized Gerchberg-Saxton algorithm. 
The Gerchberg-Saxton algorithm is commonly used for phase retrieval [43]. It requires the capture of two intensity distributions on two planes. The first is the intensity distribution at the optical field to be measured and the second is its far-field diffraction distribution. Here, we propose a simple extension of the Gerchberg-Saxton algorithm. After propagating a field through an optical system (free space can also be seen as an optical system), the corresponding output field is obtained. The intensity distributions of the input and output fields can be measured. At the same time, a virtual optical system can be simulated and modeled based on a real system.

First, the corresponding amplitude from the measured input optical intensity is assigned to the field $E_{i n}$, and its phase distribution is initialed as zero or random. Then, the incident field $E_{\text {in }}$ is propagated through the virtual optical system to get its output field $E_{\text {out }}$. The amplitude of $E_{\text {out }}$ is then replaced with the amplitude that corresponds to the measured output intensity but retains the phase of $E_{\text {out. }}$ The field $E_{\text {out. }}$ is then backpropagated through the virtual system to get the corresponding input field. The amplitude of $E_{\text {in }}$ is then replaced with the corresponding measured input intensity, but the phase is kept as the calculated one from the backpropagation. Repeating the above process can produce an optical field distribution such that the incident and output light intensities are consistent with the measured distribution. Then the wavefront detection is completed and the optical field is our result. Figure 22 shows a simulation of a generalized Gerchberg-Saxton algorithm. The optical system is free space diffraction and the distances between the input and output planes vary. When the diffraction distance is too small, the diffraction pattern contains insufficient diffraction information and the correct distribution cannot be obtained. When the diffraction distance is too large, the detector does not receive all information, and details are lost. The result is a blurred distribution.
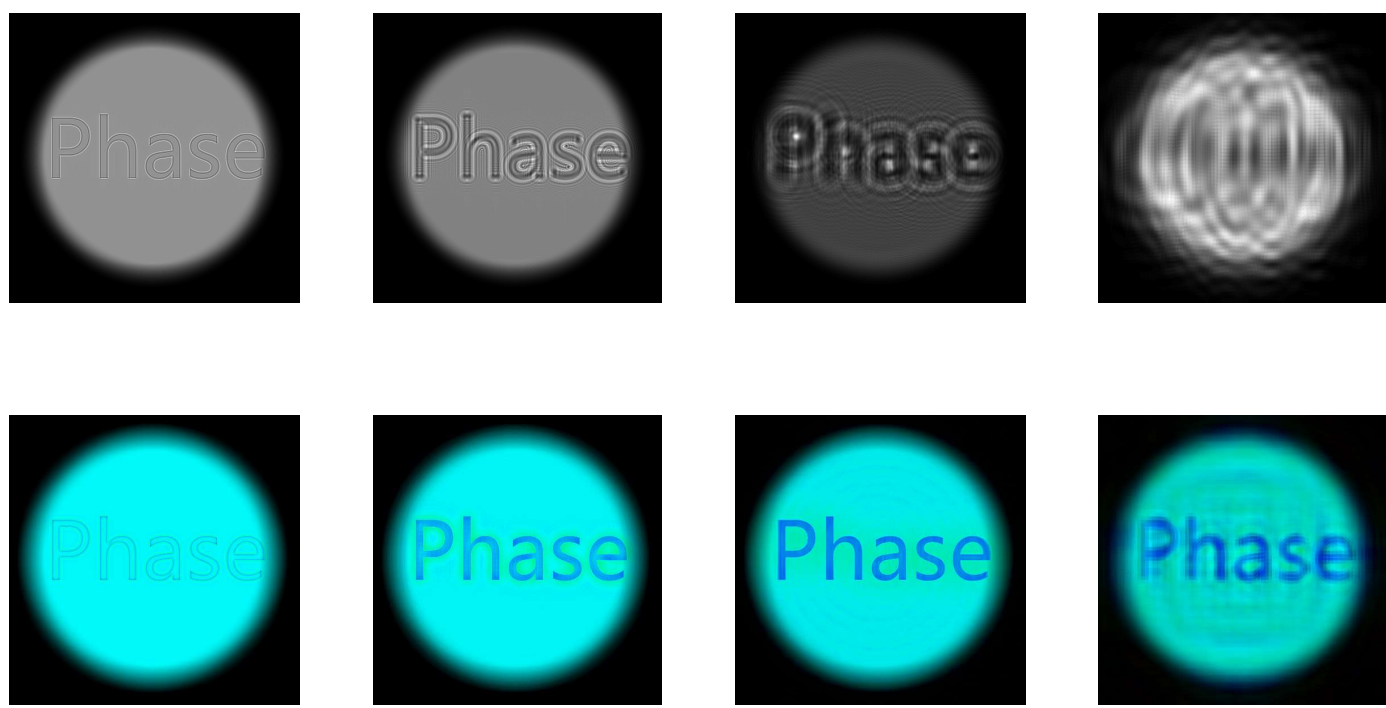

Fig. 22. Simulation of a generalized Gerchberg-Saxton algorithm.

\section{Further exploration}

In addition to the above-mentioned techniques, the typical qualitative wavefront imaging also includes spiral phase contrast imaging [44], differential interference contrast imaging [45], Dodt gradient contrast imaging [46], and oblique contrast imaging [47]. Spiral phase contrast imaging reveals the boundaries of phase objects through edge enhancement, and differential interference contrast imaging shows the gradient along one direction. Typical quantitative wavefront detection techniques also include curvature sensing [48] and pyramid wavefront sensor [49]. The foundation of curvature sensing is mainly based on the transport of intensity equation which is also important in quantitative phase imaging [50, 51]. If there are readers interested in this field, the review by Zuo [52] is recommended to read. Quantitative phase imaging and quantitative wavefront imaging are two closely related fields. For wavefront sensing, its target is the phase distribution and even the amplitude distribution of the beam on a plane, and what it cares about is the wavefront characteristics of the beam itself. But the phase in the quantitative phase imaging refers to the 
phase modulation to the light by the object, so this phase is a characteristic of the specimen to be tested, although this characteristic is related to the wavelength of the illumination light. What quantitative phase imaging really cares about is the specimen. If uniform parallel light is used to illuminate the specimen, obviously, the phase in the quantitative phase imaging is the same as the phase detected in the wavefront detection. However, in quantitative phase imaging, the relationship between the illumination light and the specimen can be changed. The most typical systems are ptychography [53-55] and Fourier ptychography $[56,57]$. Ptychography uses probe light to scan the specimen. Neighboring probes need to be partially overlapped to help reconstruct the phase. Fourier ptychography uses different illumination from a different angle, which can be taken as scanning in the spatial spectrum domain. In general, they are through multiple measurements to obtain high-resolution images but also to have a large field of view.

As for wavefront reconstruction, there is actually no lack of multiple measurement methods. Such as, Astigmatic diffraction imaging [58], RADI [8], IBRS [59] and WISH [60]. They have all measured a beam passing through different known modulations from the under-test wavefront. These technologies can be understood using the same framework as shown in Fig.23. It is similar to the generalized Gerchberg-Saxton algorithm. This kind of multiple measurement methods does not need to measure the intensity distribution of the input. But have more than one output intensity for constraint. Since these systems can introduce noncircularly symmetric. Thus, an optical vortex with singular points can be detected, which is difficult for the general diffraction imaging with Gerchberg-Saxton algorithm. At the same time, because more constraints are introduced, the iterative algorithm can be driven to converge faster.

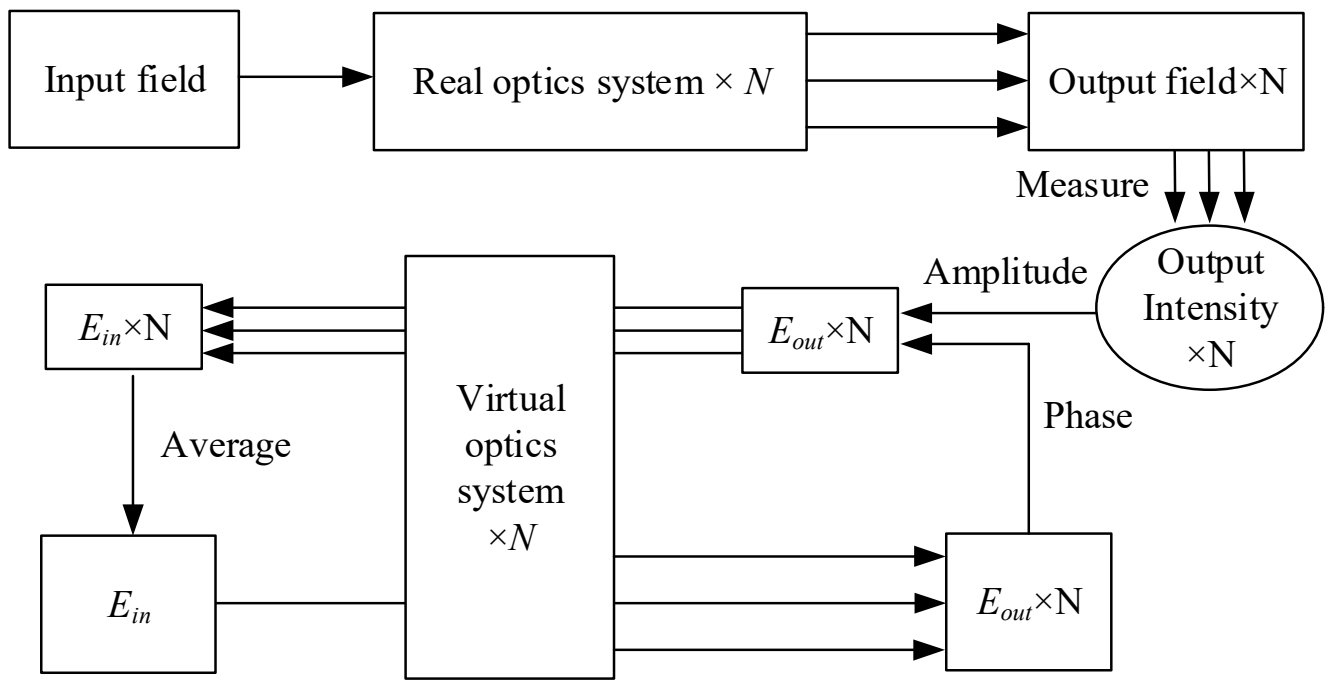

Fig. 23. Multiple measurement methods ( $\mathrm{N}$ is the number of modulation).

\section{Summary and outlook}

In this tutorial, the wavefront definition used in wavefront technology was given and we divided wavefront detection technologies into qualitative and quantitative categories. In each category, typical systems are discussed and simulated to provide a visual representation of the phase construction of that category. Different wavefront detection technologies have a specific application. They often trade-offs between parameters such as spatial resolution, response speed, and phase accuracy. Therefore, we need to select the corresponding wavefront detection technology for the specific application. For example, in adaptive optics, fast wavefront detection technology is needed. So, this area more prefers Shack-Hartmann wavefront sensor instead of the wavefront detection technology that needs an iterative algorithm for wavefront reconstruction. For situations that require human eyes to observe biological cells, phase contrast imaging is a good choice. For some case do not need high speed, but need high resolution, we can choose the phase retrieval based methods. When the input light field is not easy to obtain, multiple measurements based phase retrieval methods like WISH [60] are recommended. If observing transparent objects, the transport of intensity equation-based method is a good option. Of course, the development of high response speed, high spatial resolution, and high phase accuracy has always been the goal of this field. Current 
wavefront detection technology is aimed at monochrome scalar optical fields. To further broaden its application, wavefront detection may be extended to wavefront with polarization information, or expand to a multi-color wavefront.

Finally, we need to emphasize that the basic purpose of this article is to give readers a basic concept of wavefront and wavefront detection. The purpose of this article is not to compare current wavefront technologies, but to understand how each technology works and what information can that technology provide and use. The simulation code provided with this tutorial is used to complements this article. It provides the necessary tools for readers to better understand these technologies, and how they can be utilized and leveraged in different applications. Readers, can also use and build on top of the code to simulate the technology they are interested in, according to their own needs and specifications. This is a skill we hope readers can develop from the simulation code and the tutorial. The code on GitHub will be maintained and expanded as time goes by, by the author of this paper and the collaborations from readers and colleagues working in different fields and exploring new technologies. Also, readers are welcome to submit their code related to the non-included wavefront detection. Readers are encouraged to ask questions on the website about code bugs, technical issues, and anything they want to know about wavefront detection. This will help to update this article and codes so as to help more readers.

\section{References:}

1. Y. Park, C. Depeursinge, and G. Popescu, "Quantitative phase imaging in biomedicine," NAT PHOTONICS 12, 578-589 (2018).

2. T. J. DAVIS, D. GAO, T. E. GUREYEV, A. W. STEVENSON, and S. W. WILKINS, "PHASE-CONTRAST IMAGING OF WEAKLY ABSORBING MATERIALS USING HARD X-RAYS," NATURE 373, 595-598 (1995).

3. Z. Wang, L. Millet, M. Mir, H. Ding, S. Unarunotai, J. Rogers, M. U. Gillette, and G. Popescu, "Spatial light interference microscopy (SLIM)," OPT EXPRESS 19, 1016-1026 (2011).

4. K. Lee, K. Kim, J. Jung, J. Heo, S. Cho, S. Lee, G. Chang, Y. Jo, H. Park, and Y. Park, "Quantitative Phase Imaging Techniques for the Study of Cell Pathophysiology: From Principles to Applications," SENSORS-BASEL 13, 4170-4191 (2013). 5. T. Kim, S. Sridharan, and G. Popescu, "Gradient field microscopy of unstained specimens," OPT EXPRESS 20, 6737-6745 (2012).

6. D. Shapiro, P. Thibault, T. Beetz, V. Elser, M. Howells, C. Jacobsen, J. Kirz, E. Lima, H. Miao, A. M. Neiman, and D. Sayre, "Biological imaging by soft x-ray diffraction microscopy," Proc Natl Acad Sci U S A 102, 15343-15346 (2005).

7. M. Holler, M. Guizar-Sicairos, E. H. R. Tsai, R. Dinapoli, E. Müller, O. Bunk, J. Raabe, and G. Aeppli, "High-resolution non-destructive three-dimensional imaging of integrated circuits," NATURE 543, 402-406 (2017).

8. S. Zheng, H. Shangguan, X. Zeng, Y. Li, X. Lu, S. Xu, and D. Fan, "Coherent diffractive imaging via a rotatable cylindrical lens," OPT LASER ENG 124, 105820 (2020).

9. J. M. BECKERS, "ADAPTIVE OPTICS FOR ASTRONOMY - PRINCIPLES, PERFORMANCE, AND APPLICATIONS," ANNU REV ASTRON ASTR 31, 13-62 (1993).

10. M. J. Booth, A. A. N. Mark, R. Juškaitis, and T. Wilson, "Adaptive aberration correction in a confocal microscope," Proceedings of the National Academy of Sciences - PNAS 99, 5788-5792 (2002).

11. O. Albert, L. Sherman, G. Mourou, T. B. Norris, and G. Vdovin, "Smart microscope: an adaptive optics learning system for aberration correction in multiphoton confocal microscopy," OPT LETT 25, 52-54 (2000).

12. A. A. Malyutin, and V. A. Ilyukhin, "Generation of high-order Hermite - Gaussian modes in a flashlamp-pumped neodymium phosphate glass laser and their conversion to Laguerre - Gaussian modes," QUANTUM ELECTRON+ 37, 181-186 (2007).

13. D. Naidoo, K. Aït-Ameur, M. Brunel, and A. Forbes, "Intra-cavity generation of superpositions of Laguerre - Gaussian beams," Applied Physics B 106, 683-690 (2012).

14. S. Zheng, Z. Chen, Q. Lin, Y. Cai, X. Lu, Y. Gao, S. Xu, and D. Fan, "High-gain amplification for femtosecond optical vortex with mode-control regenerative cavity," PHOTONICS RES 8, 1375 (2020).

15. https://github.com/zhengshuiqin/Wavefront-Detection-Tutorial

16. Dallas, W.J. Wave Optics. In The Optics Encyclopedia (eds T.G. Brown, K. Creath, H. Kogelnik, M.A. Kriss, J. Schmit and M.J. Weber). (2015).

17. N. Zhang, X. Zhu, J. Yang, X. Wang, and M. Wang, "Time-resolved shadowgraphs of material ejection in intense femtosecond laser ablation of aluminum," PHYS REV LETT 99 (2007).

18. S. Rasenat, G. Hartung, B. L. Winkler, and I. Rehberg, "The shadowgraph method in convection experiments," EXP FLUIDS 7, 412-420 (1989).

19. G. S. Settles, and M. J. Hargather, "A review of recent developments in schlieren and shadowgraph techniques," Measurement science \& technology 28, 42001 (2017).

20. H. FIEDLER, K. NOTTMEYER, P. P. WEGENER, and S. RAGHU, "SCHLIEREN PHOTOGRAPHY OF WATERFLOW," EXP FLUIDS 3, 145-151 (1985). 
21. J. W. Bradley, J. Oh, O. T. Olabanji, C. Hale, R. Mariani, and K. Kontis, "Schlieren Photography of the Outflow From a Plasma Jet," IEEE T PLASMA SCI 39, 2312-2313 (2011).

22. A. Martínez-González, D. Moreno-Hernández, and J. A. Guerrero-Viramontes, "Measurement of temperature and velocity fields in a convective fluid flow in air using schlieren images," Applied optics. Optical technology and biomedical optics 52, $5562(2013)$.

23. F. Zernike, "Phase contrast, a new method for the microsopic observation of transparent objects," PHYSICA 9, 686-698 (1942).

24. Y. Fukuda, Y. Fukazawa, R. Danev, R. Shigemoto, and K. Nagayama, "Tuning of the Zernike phase-plate for visualization of detailed ultrastructure in complex biological specimens," J STRUCT BIOL 168, 476-484 (2009).

25. I. Vartiainen, M. Warmer, D. Goeries, E. Herker, R. Reimer, C. David, and A. Meents, "Towards tender X-rays with Zernike phase-contrast imaging of biological samples at $50 \mathrm{~nm}$ resolution," J SYNCHROTRON RADIAT 21, 790-794 (2014).

26. G. Popescu, T. Ikeda, R. R. Dasari, and M. S. Feld, "Diffraction phase microscopy for quantifying cell structure and dynamics," OPT LETT 31, 775-777 (2006).

27. K. Lee, K. Kim, J. Jung, J. Heo, S. Cho, S. Lee, G. Chang, Y. Jo, H. Park, and Y. Park, "Quantitative Phase Imaging Techniques for the Study of Cell Pathophysiology: From Principles to Applications," SENSORS-BASEL 13, 4170-4191 (2013). 28. Y. Sung, W. Choi, C. Fang-Yen, K. Badizadegan, R. R. Dasari, and M. S. Feld, "Optical diffraction tomography for high resolution live cell imaging," OPT EXPRESS 17, 266-277 (2009).

29. B. Bhaduri, H. Pham, M. Mir, and G. Popescu, "Diffraction phase microscopy with white light," OPT LETT 37, $1094-1096$ (2012).

30. N. Gu, Z. Yang, L. Huang, and C. Rao, "Measurement method of the misalignment between the Hartmann - Shack sensor and the deformable mirror in an adaptive optics system," J OPTICS-UK 12, 95504 (2010).

31. J. P. Veran, and G. Herriot, "Centroid gain compensation in Shack-Hartmann adaptive optics systems with natural or laser guide star," Journal of the Optical Society of America. A, Optics, image science, and vision 17, 1430 (2000).

32. J. W. Cha, J. Ballesta, and P. T. So, "Shack-Hartmann wavefront-sensor-based adaptive optics system for multiphoton microscopy," J BIOMED OPT 15, 46022 (2010).

33. L. Gilles, and B. L. Ellerbroek, "Real-time turbulence profiling with a pair of laser guide star Shack-Hartmann wavefront sensors for wide-field adaptive optics systems on large to extremely large telescopes," J Opt Soc Am A Opt Image Sci Vis 27, A76-A83 (2010).

34. Y. Liu, M. Seaberg, D. Zhu, J. Krzywinski, F. Seiboth, C. Hardin, D. Cocco, A. Aquila, B. Nagler, H. J. Lee, S. Boutet, Y. Feng, Y. Ding, G. Marcus, A. Sakdinawat, and M. P. C. U. SLAC National Accelerator Lab., "High-accuracy wavefront sensing for x-ray free electron lasers," OPTICA 5, 967 (2018).

35. P. Bon, G. Maucort, B. Wattellier, and S. Monneret, "Quadriwave lateral shearing interferometry for quantitative phase microscopy of living cells," OPT EXPRESS 17, 13080-13094 (2009).

36. P. Bon, J. Savatier, M. Merlin, B. Wattellier, and S. Monneret, "Optical detection and measurement of living cell morphometric features with single-shot quantitative phase microscopy," J BIOMED OPT 17 (2012).

37. S. Aknoun, P. Bon, J. Savatier, B. Wattellier, and S. Monneret, "Quantitative retardance imaging of biological samples using quadriwave lateral shearing interferometry," OPT EXPRESS 23, 16383 (2015).

38. S. Velghe, J. Primot, N. Guerineau, M. Cohen, and B. Wattellier, "Wave-front reconstruction from multidirectional phase derivatives generated by multilateral shearing interferometers," OPT LETT 30, 245-247 (2005).

39. H. Jiang, C. Song, C. C. Chen, R. Xu, K. S. Raines, B. P. Fahimian, C. H. Lu, T. K. Lee, A. Nakashima, J. Urano, T. Ishikawa, F. Tamanoi, and J. Miao, "Quantitative 3D imaging of whole, unstained cells by using X-ray diffraction microscopy," Proceedings of the National Academy of Sciences 107, 11234-11239 (2010).

40. K. Giewekemeyer, P. Thibault, S. Kalbfleisch, A. Beerlink, C. M. Kewish, M. Dierolf, F. Pfeiffer, and T. Salditt, "Quantitative biological imaging by ptychographic x-ray diffraction microscopy," Proceedings of the National Academy of Sciences 107, 529-534 (2010).

41. B. Abbey, G. J. Williams, M. A. Pfeifer, J. N. Clark, C. T. Putkunz, A. Torrance, I. McNulty, T. M. Levin, A. G. Peele, and K. A. Nugent, "Quantitative coherent diffractive imaging of an integrated circuit at a spatial resolution of $20 \mathrm{~nm}$," APPL PHYS LETT 93, 214101 (2008).

42. J. M. Zuo, "Atomic Resolution Imaging of a Carbon Nanotube from Diffraction Intensities," SCIENCE 300, 1419-1421 (2003).

43. G. Z. Yang, B. Z. Dong, B. Y. Gu, J. Y. Zhuang, and O. K. Ersoy, "Gerchberg-Saxton and Yang-Gu algorithms for phase retrieval in a nonunitary transform system: a comparison," Appl Opt 33, 209-218 (1994).

44. S. Fürhapter, A. Jesacher, S. Bernet, and M. Ritsch-Marte, "Spiral phase contrast imaging in microscopy," OPT EXPRESS 13, 689-694 (2005).

45. H. Ooki, Y. Iwasaki, and J. Iwasaki, "Differential interference contrast microscope with differential detection for optimizing image contrast," APPL OPTICS 35, 2230 (1996).

46. https://www.scientifica.uk.com/learning-zone/dodt-gradient-contrast

47. https://www.scientifica.uk.com/learning-zone/a-guide-to-oblique-contrast

48. F. Roddier, "Curvature Sensing And Compensation - A New Concept In Adaptive Optics," Appl Optics 27, 1223-1225 (1988). 49. S. Esposito, and A. Riccardi, "Pyramid Wavefront Sensor behavior in partial correction AdaptiveOptic systems," ASTRON ASTROPHYS 369, L9-L12 (2001).

50. C. Zuo, Q. Chen, and A. Asundi, "Boundary-artifact-free phase retrieval with the transport of intensity equation: fast solution 
with use of discrete cosine transform," OPT EXPRESS 22, 9220 (2014).

51. C. Zuo, J. Sun, J. Li, J. Zhang, A. Asundi, and Q. Chen, "High-resolution transport-of-intensity quantitative phase microscopy with annular illumination," SCI REP-UK 7 (2017).

52. C. Zuo, J. Li, J. Sun, Y. Fan, J. Zhang, L. Lu, R. Zhang, B. Wang, L. Huang, and Q. Chen, "Transport of intensity equation: a tutorial," OPT LASER ENG, 106187 (2020).

53. H. M. Faulkner, and J. M. Rodenburg, "Movable aperture lensless transmission microscopy: a novel phase retrieval algorithm," PHYS REV LETT 93, 23903 (2004).

54. J. M. Rodenburg, A. C. Hurst, A. G. Cullis, B. R. Dobson, F. Pfeiffer, O. Bunk, C. David, K. Jefimovs, and I. Johnson, "Hardx-ray lensless imaging of extended objects," PHYS REV LETT 98, 34801 (2007).

55. A. M. Maiden, J. M. Rodenburg, and M. J. Humphry, "Optical ptychography: a practical implementation with useful resolution," OPT LETT 35, 2585-2587 (2010).

56. G. Zheng, R. Horstmeyer, and C. Yang, "Wide-field, high-resolution Fourier ptychographic microscopy," NAT PHOTONICS 7, 739-745 (2013).

57. C. Zuo, J. Sun, J. Li, A. Asundi, and Q. Chen, "Wide-field high-resolution 3D microscopy with Fourier ptychographic diffraction tomography," OPT LASER ENG 128, 106003 (2020).

58. C. A. Henderson, G. J. Williams, A. G. Peele, H. M. Quiney, and K. A. Nugent, "Astigmatic phase retrieval: an experimental demonstration," OPT EXPRESS 17, 11905-11915 (2009).

59. B. Y. Wang, L. Han, Y. Yang, Q. Y. Yue, and C. S. Guo, "Wavefront sensing based on a spatial light modulator and incremental binary random sampling," OPT LETT 42, 603-606 (2017).

60. Y. Wu, M. K. Sharma, and A. Veeraraghavan, "WISH: wavefront imaging sensor with high resolution," Light: Science \& Applications 8 (2019). 